\title{
The complete mitochondrial genome of the grooved carpet shell, Ruditapes decussatus (Bivalvia, Veneridae)
}

\author{
Fabrizio Ghiselli ${ }^{\text {Corresp., }}$ 1 , Liliana Milani ${ }^{1}$, Mariangela Iannello ${ }^{1}$, Emanuele Procopio ${ }^{1}$, Peter L Chang ${ }^{2}$, \\ Sergey V Nuzhdin ${ }^{2}$, Marco Passamonti \\ 1 Department of Biological, Geological and Environmental Sciences, University of Bologna, Italy, Bologna, BO, Italy \\ 2 Department of Biological Sciences, Program in Molecular and Computational Biology, University of Southern California, Los Angeles, CA, United States \\ Corresponding Author: Fabrizio Ghiselli \\ Email address: fabrizio.ghiselli@unibo.it
}

Despite the large number of animal complete mitochondrial genomes currently available in public databases, knowledge about mitochondrial genomics in invertebrates is uneven. This paper reports, for the first time, the complete mitochondrial genome of the grooved carpet shell, Ruditapes decussatus, also known as the European clam. $R$. decussatus is morphologically and ecologically similar to the Manila clam Ruditapes philippinarum, which has been recently introduced for aquaculture in the very same habitats of $R$. decussatus, and that is replacing the native species. Currently the production of the European clam is almost insignificant, nonetheless it is considered a high value product, and therefore it is an economically important species, especially in Portugal, Spain and Italy. In this work we: i) assembled $R$. decussatus mitochondrial genome from RNA-Seq data, and validated it by Sanger sequencing, ii) analyzed and characterized the $R$. decussatus mitochondrial genome, comparing its features with those of other venerid bivalves; iii) assessed mitochondrial sequence polymorphism (SP) and copy number variation (CNV) of tandem repeats across 26 samples. Despite using high-throughput approaches we did not find evidence for the presence of two sex-linked mitochondrial genomes, typical of the Doubly Uniparental Inheritance of mitochondria, a phenomenon known in 100 bivalve species. According to our analyses, $R$. decussatus is more genetically similar to species of the Genus Paphia than to the congeneric $R$. philippinarum, a finding that bolsters the alreadyproposed need of a taxonomic revision. We also found a quite low genetic variability across the examined samples, with few SPs and little variability of the sequences flanking the control region (Largest Unassigned Regions, LURs). Strikingly, although we found low nucleotide variability along the entire mitochondrial genome, we observed high levels of length polymorphism in the LUR due to CNV of tandem repeats, and even a LUR length heteroplasmy in two samples. It is not clear if the lack of genetic variability in the mitochondrial genome of $R$. decussatus is a cause or an effect of the ongoing replacement 
of $R$. decussatus with the invasive $R$. philippinarum, and more analyses, especially on nuclear sequences, are required to assess this point. 


\section{AUTHOR COVER PAGE}

2

3 The Complete Mitochondrial Genome of the Grooved Carpet Shell, Ruditapes decussatus

4 (Bivalvia, Veneridae).

5

6 Fabrizio Ghiselli ${ }^{1, *,}{ }^{\circ}$, Liliana Milani ${ }^{1,}{ }^{\circ}$, Mariangela Iannello ${ }^{1}$, Emanuele Procopio ${ }^{1}$, Peter L.

7 Chang ${ }^{2}$, Sergey V. Nuzhdin ${ }^{2}$, and Marco Passamonti ${ }^{1}$

8

$9{ }^{1}$ Department of Biological, Geological and Environmental Sciences - University of Bologna, 10 Italy.

112 Program in Molecular and Computational Biology, Department of Biological Sciences,

12 University of Southern California, Los Angeles, CA, USA.

13

$14{ }^{\circ}$ Equal contribution

15

16

17

18

19

20

21

22

$23 *$ Corresponding Author: Fabrizio Ghiselli fabrizio.ghiselli@unibo.it 


\section{Abstract}

25 Despite the large number of animal complete mitochondrial genomes currently available in public databases, knowledge about mitochondrial genomics in invertebrates is uneven. This paper reports, for the first time, the complete mitochondrial genome of the grooved carpet shell, Ruditapes decussatus, also known as the European clam. $R$. decussatus is morphologically and ecologically similar to the Manila clam Ruditapes philippinarum, which has been recently introduced for aquaculture in the very same habitats of $R$. decussatus, and that is replacing the native species. Currently the production of the European clam is almost insignificant, nonetheless it is considered a high value product, and therefore it is an economically important species, especially in Portugal, Spain and Italy.

In this work we: $i$ ) assembled $R$. decussatus mitochondrial genome from RNA-Seq data, and genome, comparing its features with those of other venerid bivalves; iii) assessed mitochondrial sequence polymorphism (SP) and copy number variation (CNV) of tandem repeats across 26 samples.

Despite using high-throughput approaches we did not find evidence for the presence of two sexlinked mitochondrial genomes, typical of the Doubly Uniparental Inheritance of mitochondria, a phenomenon known in $\sim 100$ bivalve species. According to our analyses, $R$. decussatus is more genetically similar to species of the Genus Paphia than to the congeneric $R$. philippinarum, a

43 finding that bolsters the already-proposed need of a taxonomic revision. We also found a quite 44 low genetic variability across the examined samples, with few SPs and little variability of the sequences flanking the control region (Largest Unassigned Regions, LURs). Strikingly, although we found low nucleotide variability along the entire mitochondrial genome, we observed high 
47 levels of length polymorphism in the LUR due to CNV of tandem repeats, and even a LUR

48 length heteroplasmy in two samples. It is not clear if the lack of genetic variability in the

49 mitochondrial genome of $R$. decussatus is a cause or an effect of the ongoing replacement of $R$.

50 decussatus with the invasive $R$. philippinarum, and more analyses, especially on nuclear

51 sequences, are required to assess this point.

52

53

54

\section{Keywords: (3-10)}

56 complete mitochondrial genome; mitochondrial length polymorphism; mitochondrial repeats;

57 codon usage; bivalve molluscs; European clam; comparative mitochondrial genomics; Doubly

58 Uniparental Inheritance; mtDNA de novo assembly; RNA-Seq.

59 


\section{Introduction}

1

Despite a large number of animal complete mitochondrial genomes (mtDNAs) being available in public databases ( $>55,000$ in GenBank), up to now sequencing has been focused mostly on vertebrates ( 50,000 in GenBank), and the current knowledge about mitochondrial genomics in invertebrates — with the notable exception of few model organisms (e.g. Drosophila and Caenorhabditis elegans) — is uneven. To better understand invertebrate mitochondrial biologyand, most importantly, mitochondrial biology and evolution in general — it is necessary to adopt a more widespread approach in gathering and analyzing data. Failing to do so would bias our knowledge toward a few taxonomic groups, with the risk of losing a big part of the molecular and functional diversity of mitochondria. Actually, despite maintaining its core features in terms of genetic content, mtDNA in Metazoa shows a wide range of variation in some other traits such as, for example, genome architecture, abundance of unassigned regions (URs) — namely regions with no assigned product (protein, RNA) — repeat content, gene duplications, introns, UTRs, and even additional coding genes (see Breton et al., 2014 for a review) or genetic elements (e.g, small RNAs, see Pozzi et al., 2017) . All this emerging diversity is in sharp contrast with the —at this point outdated - textbook notion about mtDNAs role being limited to the production of a few subunits of the protein complexes involved in oxidative phosphorylation (OXPHOS). This paper reports, for the first time, the complete mitochondrial genome of the grooved carpet shell, Ruditapes decussatus (Linnaeus, 1758). R. decussatus-also known as the European clam - is distributed all over the Mediterranean coasts, as well as on the Atlantic shores, from Lofoten Islands (Norway) to Mauritania, including the British Isles. R. decussatus lives in warm coastal waters, especially in lagoons, and it is morphologically and ecologically similar to the 
83 Manila clam Ruditapes philippinarum, which has been recently introduced for aquaculture in the

84 very same habitats of $R$. decussatus. $R$. philippinarum, native from the Philippines, Korea, and

85 Japan, was accidentally introduced into North America in the 1930s, and from there it was

86 purposely introduced in France (1972), UK (1980), and Ireland (1982) for aquaculture purposes

87 (Gosling, 2003). According to historical records, R. decussatus was one of the most important

88 species for aquaculture in Europe, but overfishing, irregular yields, recruitment failure, and

89 outbreaks of bacterial infection pushed the producers to introduce $R$. philippinarum; Italy

90 imported large quantities of $R$. philippinarum seed from UK in 1983 and 1984. Compared to the

91 European clam, the Manila clam turned out to be faster growing, more resistant to disease, to

92 have a more extended breeding period and a greater number of spawning events, and to begin

93 sexual maturation earlier (i.e. at a smaller size). Upon introduction of the more robust $R$.

94 philippinarum, $R$. decussatus suffered a population decline in the Southwestern Europe (Arias-

95 Pérez et al., 2015), and currently the production of the European clam is almost insignificant.

96 Nonetheless the grooved carpet shell is considered a high value product, and therefore it is an

97 economically important species, especially in Portugal, Spain and Italy (Gosling, 2003; Leite et

98 al., 2013; de Sousa et al., 2014).

99 Molluscs in general, and bivalves in particular, exhibit an extraordinary degree of mtDNA

100 variability and unusual features, such as: large mitochondrial genomes (up to $\sim 47 \mathrm{~Kb}$ ), high

101 proportion of URs (i.e. number of base pairs annotated as URs over the total mtDNA length),

102 novel protein coding genes with unknown function, frequent and extensive gene rearrangement,

103 and differences in strand usage (Gissi, Iannelli \& Pesole, 2008; Breton et al., 2011; Ghiselli et

104 al., 2013; Milani et al., 2014b; Plazzi, Puccio \& Passamonti, 2016). Moreover, mitochondrial

105 genome size varies among bivalves because of gene duplications and losses (Serb \& Lydeard, 
106 2003; Passamonti et al., 2011; Ghiselli et al., 2013), and sometimes genes are fragmented as in

107 the case of ribosomal genes in oysters (Milbury et al., 2010). The most notable feature of bivalve

108 mtDNA is the Doubly Uniparental Inheritance (DUI) system of transmission (Skibinski,

109 Gallagher \& Beynon, 1994a,b; Zouros et al., 1994a,b). Under DUI, two different mitochondrial

110 lineages (and their respective genomes) are transmitted to the progeny: one is inherited from the

111 egg (female-transmitted or F-type mtDNA), the other is inherited from the spermatozoon (male-

112 transmitted or M-type mtDNA). Following fertilization, the early embryo is heteroplasmic, but

113 the type of mitochondria present in the adult is tightly linked to its sex. Females are commonly

114 homoplasmic for $\mathrm{F}$, while males are heteroplasmic with the following distribution of mtDNA

115 types: the germ line is homoplasmic for the M-type (which will be transmitted via sperm to male

116 progeny), the soma is heteroplasmic to various degrees, depending on tissue type and/or species

117 (Ghiselli, Milani \& Passamonti, 2011; Zouros, 2013). To date, the only known animals

118 exhibiting DUI are about 100 species of bivalve molluscs (Gusman et al., 2016). This natural and

119 evolutionarily stable heteroplasmic system can be extremely useful to investigate several aspects

120 of mitochondrial biology (see Passamonti \& Ghiselli, 2009; Breton et al., 2014; Milani \&

121 Ghiselli, 2015; Milani, Ghiselli \& Passamonti, 2016). Indeed, despite the fact that many aspects

122 of DUI are still unknown, there is evidence that DUI evolved from a strictly maternal inheritance

123 (SMI) system (Milani \& Ghiselli, 2015; Milani, Ghiselli \& Passamonti, 2016), by modifications

124 of the molecular machinery involved in mitochondrial inheritance, through as-yet-unknown

125 specific factors (see Diz, Dudley \& Skibinski, 2012; and Zouros, 2013 for proposed models).

126 The detection of DUI is not a straightforward process, especially using PCR-based approaches:

127 given that the divergence between $\mathrm{F}$ and $\mathrm{M}$ genomes is often comparable to the distance between

128 mtDNAs of different classes of Vertebrates, primers may fail to amplify one of the two mtDNAs, 
129 yielding a false-negative result. Moreover, M-type mtDNA can be rare in somatic tissues, so it

130 may be difficult to amplify from animals sampled outside of the reproductive season, when

131 gonads are absent (thoroughly discussed in Theologidis et al., 2008). High-throughput

132 sequencing (HTS) approaches can overcome such problems, because a prior knowledge of the

133 mtDNA sequence is not needed, and low-copy variants can be easily unveiled (see for example:

134 Ju et al. 2011; King et al. 2014). Until now, HTS has been scarcely utilized to study

135 mitochondrial transcriptomes and genomes (Pesole et al., 2012; Smith, 2013), even if it showed

136 very good potential (Lubośny et al., 2017/2; see for example Yuan et al., 2016). In this work we:

137 i) assembled $R$. decussatus mitochondrial genome from RNA-Seq data, and validated it by

138 Sanger sequencing, ii) analyzed and characterized $R$. decussatus mitochondrial genome,

139 comparing its features with those of other venerid bivalves; $i i i)$ assessed mitochondrial sequence

140 polymorphism (SP) and structural variants - copy number variation (CNV) of tandem repeats-

141 among the sampled animals.

142

143 Materials \& Methods

144

145 Sampling

146 The 26 Ruditapes decussatus specimens used in this study were collected from the Northern

147 Adriatic Sea, in the river Po delta region (Sacca di Goro, approximate GPS coordinates:

$14844^{\circ} 50^{\prime} 06^{\prime} \mathrm{N}, 12^{\circ} 17^{\prime} 55^{\prime} \mathrm{E}$ ) at the end of July 2011, during the spawning season. Each individual

149 was dissected, and gonadal liquid collected with a glass capillary tube. All the samples showed

150 ripe gonads, consistently with the time of the year when the sampling occurred. The gonadal

151 liquid was checked under a light microscope to assess the sex of the individual, and to make sure 
152 that the sample consisted of mature gametes. Both the gamete samples and the clam bodies were

153 flash-frozen in liquid nitrogen, and stored at $-80^{\circ} \mathrm{C}$, until nucleic acid extraction. Supplementary

154 Table 1 shows the sample list, and details about data availability.

155

156

157

158

159

160

161

162

163

164 165 as well.

RNA-Seq reads.

In total, 12 samples (6 males and 6 females) were used for RNA-Seq. Total RNA extraction and library preparation were performed following the protocol described in Mortazavi et al. (2008), with the modifications specified in Ghiselli et al. (2012). The 12 samples were indexed, pooled and sequenced in two lanes (two technical replicates) of Illumina GA IIx, using 76bp paired-end

\section{De Novo Assembly}

The mitochondrial genome of $R$. decussatus was not available in the databases, so we used the transcriptome data to generate a draft to be used as a guide for Sanger sequencing. Illumina reads from all 12 samples were pooled and compared to a set of 20 bivalvia mitochondrial genomes to identify reads with mitochondrial origin. Alignment was done using BLASTN. All reads with similarity yielding E-value $<1 \mathrm{E}-5$ were then assembled into contigs using the A5 pipeline (version 2013032; Tritt et al, 2012) and joined into scaffolds using CAP3 (Huang \& Madan, 1999). For the quality check step, we applied a PHRED Q-score cutoff threshold of 33; the other A5 parameters were set as default. CAP3 was run with default settings 


\section{Sanger Validation}

175 In total, $14 R$. decussatus samples from the same collection campaign — sexed, and stored at $17680^{\circ} \mathrm{C}$ - were used for DNA extraction. DNA from the gonadic tissue was extracted using the 177 Qiagen DNeasy kit. Primers for mtDNA amplification were designed based on contigs obtained 178 from RNA-Seq matching venerid mtDNA sequences, then the "primer walking" method was 179 used to Sanger-sequence the complete mitochondrial genome of $R$. decussatus. The primers were 180 designed with the software Primer3 (Rozen \& Skaletsky, 2000) and tested on several samples, 181 then a female was chosen as reference sample for Sanger validation of mtDNA de novo 182 assembly. In addition, we amplified the Largest Unassigned Region (LUR) of 13 females to 183 assess its variability (see Results and Discussion). The list of the primers and their sequences are 184 reported in Supplementary Table 2. PCR reactions were performed in a final volume of $50 \mu 1$ 185 using the GoTaq Flexi DNA Polymerase Kit (Promega), on a 2720 Thermal Cycler (Applied 186 Biosystem). The PCR reactions were set as follows: initial denaturation $95^{\circ} \mathrm{C}$ for 1 min, then 30 187 cycles of amplification (denaturation $95^{\circ} \mathrm{C}$ for $1 \mathrm{~min}$, annealing $48-60^{\circ} \mathrm{C}$ for $1 \mathrm{~min}$, extension $18872^{\circ} \mathrm{C}$ for $1 \mathrm{~min} / \mathrm{kb}$ ), then the final extension at $72^{\circ} \mathrm{C}$ for $5 \mathrm{~min}$. PCR products were checked by 189 electrophoretic run on 1\% agarose gel, and then purified using the DNA Clean \& Concentrator19025 kit (Zymo Research).

191 Sanger sequencing was performed by Macrogen Inc. (http://www.macrogen.com).

192 Sequences were aligned with the software MEGA 6.0 (Tamura et al., 2013), using the contigs 193 obtained by RNA-seq as a reference. 
196 Open Reading Frames (ORFs) were identified with ORF finder (Wheeler et al., 2005).

197 Alternative start codons were considered functional because they are common in Bivalvia. ORFs

198 were annotated starting from the first available start codon (ATG, ATA or ATC) downstream of

199 the preceding gene, and ending with the first stop codon in frame (TAA or TAG). tRNA genes

200 and their structure were identified with MITOS (Bernt et al., 2013) and ARWEN (Laslett \&

201 Canback, 2008). Secondary structures were predicted using the RNAFold Server, included in the

202 ViennaRNA Web Services (http://rna.tbi.univie.ac.at/; Gruber et al., 2008); the folding

203 temperature was set at $16^{\circ} \mathrm{C}$ which is the average annual temperature of the water from which the

$204 R$. decussatus specimens used in this work were fished (download RNAFold results from

205 figshare: https://ndownloader.figshare.com/files/8387672). tRNAs and other secondary

206 structures were drawn with the software Varna GUI (Darty, Denise \& Ponty, 2009). Ribosomal

207 small subunit $(r r n S)$ and large subunit $(r r n L)$ were identified with BLASTN, and annotated

208 considering the start and the end of the adjacent genes as the boundaries of the rRNA genes.

209 Non-genic regions were annotated as Unassigned Regions (URs). In order to identify the putative

210 D-loop/control region (CR), we analyzed the LUR with the MEME suite (Bailey et al., 2009) to

211 find DNA motifs using the following bivalve species as comparison: Acanthocardia tuberculata,

212 Arctica islandica, Coelomactra antiquata, Fulvia mutica, Hiatella arctica, Loripes lacteus,

213 Lucinella divaricata, Lutraria rhynchaena, Meretrix lamarckii (F-type), Meretrix lamarckii (M-

214 type), Meretrix lusoria, Meretrix lyrata, Meretrix meretrix, Meretrix petechialis, Moerella

215 iridescens, Nuttallia olivacea, Paphia amabilis, Paphia euglypta, Paphia textile, Paphia

216 undulata, Ruditapes philippinarum (F-type), Ruditapes philippinarum (M-type), Semele scabra,

217 Sinonovacula constricta, Solecurtus divaricatus, Solen grandis, Solen strictus, Soletellina diphos 
218 and the sea urchin Strongylocentrotus purpuratus (Echinoidea, Strongylocentrotidae). The list of

219 the species used in the phylogenetic analysis and in the comparative analyses of DNA motifs,

220 sequence similarity, and gene order are available in Supplementary Table 3. The GOMo (Gene

221 Ontology for Motifs; Buske et al., 2010) tool of the MEME suite was used to assign GO terms to 222 the motifs discovered.

223 The number of repeats in the LUR of the reference sample (F4) was calculated with tandem 224 repeat finder (http://tandem.bu.edu/trf/trf.html), since the complete LUR sequence was available 225 (download tandem repeat finder results from figshare:

$226 \mathrm{https} / / /$ ndownloader.figshare.com/files/8387666). In the other cases, in which the LUR could not 227 be sequenced without gaps, the number of repeats was inferred from agarose gel electrophoresis.

Other Analyses

230 Comparisons among venerid complete mtDNAs were performed with BLAST Ring Image 231 Generator (BRIG, Alikhan et al., 2011) and Easyfig (Sullivan, Petty \& Beatson, 2011).

232 Descriptive statistics were obtained with MEGA v6.0 (Tamura et al., 2013), except for the codon 233 usage table, which was obtained with the Sequence Manipulation Suite (Stothard, 2000).

234 Sequence polymorphism (SP) assessment from RNA-Seq reads was performed with the Genome 235 Analysis Toolkit (GATK, McKenna et al., 2010), with the Sanger-sequenced mtDNA as 236 reference. For SP discovery and genotyping we used standard hard filtering parameters or variant 237 quality score recalibration (DePristo et al., 2011). The MitoPhast pipeline (Tan et al., 2015) was 238 used to obtain the Maximum Likelihood (ML) tree, which was visualized with Evolview v2 (He 239 et al., 2016). Briefly, MitoPhast takes as input GenBank files (.gb), extracts the coding 240 sequences, profiles the sequences with Pfam (Finn et al., 2016) and PRINTS (Attwood et al., 
241 2003), performs a multiple sequence alignment with Clustal Omega (Sievers et al., 2011),

242 removes poorly aligned regions with trimAl (Capella-Gutiérrez, Silla-Martínez \& Gabaldón,

243 2009), concatenates the coding sequences, performs data partitioning and model selection, and

244 then carries out a ML analysis using RAxML (Stamatakis, 2014). The species used in the ML

245 analysis, and their GenBank Accession Numbers are listed in Supplementary Table 3. Amino

246 acid sequences of three different cox3 ORFs inferred from Sanger sequencing and GATK

247 polymorphism data were analyzed with InterProScan (Jones et al. 2014).

248

249 Results

250 De Novo Assembly and Sanger Validation

251 Despite using HTS on extracts of ripe gonads (i.e. mature gametes), and multiple assembly

252 strategies (see Discussion for details) we could not find evidence for DUI. The de novo assembly

253 process produced 9 contigs, of which 8 included multiple genes, and one included a single gene

254 (see Table 1). The sequences of the contigs in FASTA format are available on figshare

255 (https://ndownloader.figshare.com/files/8906839). In four cases (Contigs 1, 3, 6, and 7) a clear

256 polyadenylation signal was present, in other four cases (Contigs 2, 5, 8, and 9) it was not. Contig

257 4, the only one including a single gene ( $\operatorname{cox} 3)$, ends with just 8 As, so it is not clear if a

258 polyadenylation signal is present in this case. In Contig 7 (that includes cox2, $t R N A-I l e, n d 4 L$,

$259 n d 2, n d 1, t R N A-L e u 1$, and cox 1 genes) there is a polyadenylation signal (56 As) after the cox2

260 gene.

261 The 9 contigs were used as a scaffold for the primer walking procedure used for Sanger

262 validation of the de novo assembly. We first tried to connect the contigs designing primers close

263 to the 5' and 3' ends of each contig and pairing them following the gene order of Paphia, because 
264 the sequence of genes in the contigs suggested that $R$. decussatus gene order might have been 265 similar. During such process, Contig 1 turned out to be a chimeric assembly between two non266 contiguous portions of the mtDNA, one including atp $6, n d 3$, and $n d 5$, the other including coxl, 267 tRNA-Leul, $n d 1, n d 2$, and $n d 4 L$. Once we amplified and sequenced the portions of mtDNA 268 between the contigs, we proceeded with the Sanger resequencing of the remaining parts.

Annotation and mtDNA Features

271 The mitochondrial genome contains 13 protein-coding genes, and in the reference female is 27218,995 bp long (Figure 1); the gene arrangement and other details are shown in Table 2. All 273 genes are located on the heavy strand, and in addition to the classic start codon ATG (Met), the 274 alternative start codons ATA (Met) and ATC (Ile) are present. The most frequently used start 275 codons are: ATA (cox1, nd1, nd4L, cox2, cob, atp8, nd4), and ATG (nd2, atp6, nd3, nd5, nd6, 276 cox3). The stop codons found are TAG (coxl, nd2, nd4L, cox2, cytb, nd4) and TAA ( $n d 1$, atp6, $277 n d 3$, atp 8, $n d 6$ ). The $n d 4$ gene has an incomplete stop codon (TA-). 22 tRNA genes were 278 identified, including two tRNAs for leucine, tRNA-Leu1(TAG) and tRNA-Leu2(TAA), and two 279 for serine, tRNA-Ser1(TCT) and tRNA-Ser2(TGA), both showing degenerate D-arm branches. 280 tRNA structures are shown in Supplementary Figure 1. The two rRNAs, $r r n S$ and $r r n L$, were 281 both identified: the $r r n S$ is located between cox3 and coxl, while $r r n L$ is between cytb and atp6. 282 Unassigned Regions (URs) were identified on the basis of unannotated spaces between different 283 genes; we found 24 URs (Table 3).

284 The analysis of the nucleotide composition points out that the mitochondrial genome of this 285 bivalve species exhibits high $\mathrm{A}+\mathrm{T}$ content, totaling $63 \%$ versus $37 \% \mathrm{G}+\mathrm{C}$. The minimum values 286 of $\mathrm{A}+\mathrm{T}$ are found in cytb (60.1\%) and $n d 4(61 \%)$. The nucleotide composition of every gene is 
287 shown in Table 4. According to the analysis above, both A and T occur very frequently at the 288 third position of codons ( $64.6 \%$ on average of $\mathrm{A}+\mathrm{T})$, while the less frequent base in third 289 position is C (12\%). The most used codons are UUU (Phe), counted 269 times, and UUA (Leu) 290 counted 210 times ( $6.78 \%$ and $5.29 \%$ of the total, respectively), while the less used codons are 291 CGC (Arg) counted 6 times (0.15\%), ACC (Thr) and CCG (Pro) each counted 16 times ( $0.4 \%)$ 292 (Table 5). Only in 4 cases over 20 (Lys, Leu, Gln, Val), the most frequently used codon matches 293 the correspondent mitochondrial tRNA anticodon.

294 The UR11 is the Largest Unassigned Region (LUR) and is located between atp 8 and $n d 5$ 295 (Figures 1 and 2A). The LUR of the female used for whole mtDNA Sanger sequencing (i.e. the 296 reference female, F4) is 2,110 bp long, and includes 6.5 repeated sequences - each repeat having 297 a length of $54 \mathrm{bp}$-localized in the 3' region of the LUR, just upstream the atp 8 gene (Figure 298 2A). DNA secondary structure analysis predicted 3 stem-loop structures in such region (Figure 299 2B and supplementary files on figshare: https://ndownloader.figshare.com/files/8387672), with a 300 change in Gibbs free energy $(\Delta \mathrm{G})$ of $-71.38 \mathrm{Kcal} / \mathrm{mol}$. We also amplified and sequenced the 301 LUR of 13 more females. We were not able to completely sequence LURs longer than 2,110 bp, 302 because of the known difficulties in Sanger sequencing of regions including multiple repeats. 303 The sequence alignment of the 13 LURs is available for download from figshare 304 (https://ndownloader.figshare.com/files/8360789). LUR lengths, inferred from gel 305 electrophoresis, are reported in Table 6, and they range from 2,000 to 5,000 bp. Two females (F3 306 and F17) showed length heteroplasmy of the LUR. The portion of the genome occupied by URs 307 varies between $14.11 \%$ and $29.38 \%$, depending on LUR length. The analysis with MEME 308 (output shown in Supplementary Figures 2 and 3) unveiled two motifs (Figure 2C) that show a 309 strong conservation within the Veneridae family, and with S. purpuratus. The sea urchin was 
310 included in the analysis because Cao et al. (2004) reported a match between some motifs found

311 in the CR of the marine mussels Mytilus edulis and Mytilus galloprovincialis with regulatory

312 elements of the sea urchin CR. Accordingly, the search with GOMo assigned a series of GO

313 terms related to transcription to the two motifs (Supplementary Table 4).

314

315 Polymorphism

316 Table 7 (top) shows the statistics associated with the SP analysis performed with GATK on the

31712 samples used for RNA-Seq, with the Sanger-sequenced mtDNA as reference. Overall, 257

318 SPs were called, of which 145 (56.4\%) were located in coding sequences (CDS). Interestingly,

319 most of the SPs were called because of private alleles of one single male specimen (mRDI01).

320 More in detail, 151 SPs out of 257 (58.7\%) along the whole mtDNA sequence, and 103 SPs out

321 of $145(71 \%)$ in CDS, were private of mRDI01. In CDS, if we exclude the SPs associated with

322 this male, the number of polymorphisms drops to 42 over 14,920 bp of coding mtDNA (GATK

323 output in VCF format and a detailed list of SPs in tabular format is available on figshare:

324 https://ndownloader.figshare.com/files/8902537), of which 18 are represented by indels, 6 of

325 which are located in 4 different coding genes: one each in cox $1, c y t b$, and $n d 5$, plus 3 in cox3

326 (see Table 8). A file showing the ORF generated by the different variants of cox3, and

327 alignments between them is available on figshare

328 (https://ndownloader.figshare.com/files/8402471). Table 7 (bottom) shows the number of SPs in 329 males, in males except mRDI01, and in females both along the whole mtDNA, and in CDS. The 330 number in brackets represent the number of private SPs for each category.

331

332 Comparison with Other Veneridae 
333 Figure 3 shows the $R$. decussatus mtDNA map (external gray circle), and the BLASTN identity

334 (colored inner circles) with complete mtDNAs of other 10 venerid species (see list in

335 Supplementary Table 3). Figure 4 shows the ML tree obtained with the MitoPhast pipeline; the

336 complete input and output of this analysis is available on figshare

337 (https://ndownloader.figshare.com/files/8360792). Figure 5 shows the variation in gene order

338 between $R$. decussatus and P. euglypta (Figure 5A), M. lamarckii F-type (Figure 5B), $R$.

339 philippinarum F-type (Figure 5C), and among all the 4 species (Figure 5D).

340

341 Discussion

342 RNA-Seq-guided Sequencing of $m t D N A$

343 The de novo assembly of the mtDNA from RNA-Seq data turned out to be informative,

344 simplifying the primer walking procedure used for Sanger sequencing. Only one conting (Contig

345 1) resulted to be a chimeric sequence obtained by the misassembly of two smaller contigs. Most

346 of the contigs (8 out of 9) contained more than one gene, and most of the tRNA genes were

347 included in the de novo assembly. Except for $t R N A-P r o, t R N A-I l e$, and $t R N A-L e u 1$, all the other

348 tRNA genes are organized in two big clusters: a 13-gene cluster positioned between cox3 and

$349 n d 6$, and a 6-gene cluster between $n d 6$ and $n d 4$. The assembly retrieved 6 out of 13 tRNAs from

350 the first cluster (missing $t R N A-G l y, t R N A-G l u, t R N A-A s n, t R N A-T h r, t R N A-C y s, t R N A-A l a$, and

$351 t R N A-S e r 1$ ), and 4 out of 6 tRNAs from the second cluster (missing $t R N A-M e t$ and $t R N A-A s p$ ).

352 All the tRNA genes not located in these two clusters ( $t R N A-P r o, t R N A-I l e$, and $t R N A-L e u 1$ ) were

353 included in the contigs. The presence of a clear polyadenylation signal in 4 of the assembled

354 contigs (see Table 1) seems to indicate the existence of multiple polycistronic transcripts. It is

355 also noteworthy that poly-A sequences seem to be absent in contigs having tRNA of rRNA genes 
356 at one end (Contigs 2, 5, 8 and 9). This could be either an evidence supporting the "tRNA

357 punctation model" of RNA processing proposed by Ojala et al. (1981) for human mitochondria, 358 or a result of difficulties in sequencing/assembly of such regions. More analyses are required to 359 address this point.

360

361 General Features

362 The size of the fully Sanger-sequenced mitochondrial genome of $R$. decussatus (reference female $363 \mathrm{~F} 4$ ) is of $18,995 \mathrm{bp}$, and it includes 13 protein-coding genes, 22 tRNAs and 2 rRNAs. Our data 364 support the presence of the atp 8 gene in the mtDNA of $R$. decussatus; atp 8 has been reported as 365 missing in several bivalve species, however more accurate searches often led to the identification 366 of the gene, so, in most cases, the alleged lack of atp 8 is likely ascribable to annotation 367 inaccuracies due to the extreme variability and the small size of the gene (Breton, Stewart \& 368 Hoeh, 2010; Breton et al., 2014; Plazzi, Puccio \& Passamonti 2016).

369 The mitochondrial genome of $R$. decussatus shows a high content of A-T (63\%), a common 370 feature in bivalve mtDNAs; moreover, $\mathrm{T}$ is the most common nucleotide at the third codon base 371 (64.6\%). The most common codon is UUU (Phe), which is also the most commonly used in 372 bivalves, as well as in other invertebrates (Passamonti et al., 2011).

373

374 Codon Usage

375 As shown in Table 5, in 16 cases out of 20 , the most frequently used codon does not correspond 376 to the anticodon of the inferred tRNA. In other words, there is not a correspondence between the 377 most abundant codons and the anticodons of the 22 mitochondrial tRNAs. According to the 378 "wobble hypothesis" - first proposed by Francis Crick (1966) - the conformation of the tRNA 
379 anticodon loop enables some flexibility at the first base of the anticodon, so a Watson-Crick type

380 of base pairing in the third position of the codon is not strictly necessary. This allows an amino

381 acid to be correctly incorporated by ribosomes even if the tRNA is not fully complementary to

382 the codon; according to Crick, this explains the degeneracy of the genetic code. This feature is

383 particularly interesting in the light of the debate about natural selection acting at synonymous

384 sites: since the early 1980s, evidence of a correlation between synonymous codon usage and

385 tRNA abundances started accumulating. According to these authors, synonymous codon usage is

386 biased to match skews in tRNA abundance, as a result of selective pressure maximizing protein

387 synthesis rates (reviewed in Chamary, Parmley \& Hurst, 2006). Following this rationale, the

388 results here reported and data from other marine bivalves and metazoans ( $\mathrm{Yu} \& \mathrm{Li}, 2011$;

389 Passamonti et al., 2011) would suggest that in some mitochondrial genomes translation

390 efficiency is not maximized, and this observation deserves further investigation.

391

392

\section{Length and Sequence Polymorphism}

393 The mtDNA of $R$. decussatus has a high proportion of URs mostly depending on the length of

394 the LUR (Table 6); on average, bivalve mtDNAs have 1.7x the amount of URs in respect to

395 other analyzed Metazoa (Ghiselli et al., 2013), and it is still unclear whether there is an

396 accumulation of non-functional sequences in bivalve mtDNA due to genetic drift, or if such URs

397 are maintained by natural selection because they contain — so far unknown—functional elements

398 (see for example Milani et al., 2013, 2014b; Breton et al., 2014; Pozzi et al., 2017). The LUR of

$399 R$. decussatus most likely includes the mitochondrial CR, as indicated by the presence of two

400 motifs (Figure 2C, Supplementary Figures 3 and 4) similar to two regulatory elements identified

401 in the sea urchin CR. These two motifs are the same identified in previous analyses on the clam 
402 R. philippinarum and the mussel Musculista senhousia (Ghiselli et al., 2013; Guerra, Ghiselli \& 403 Passamonti, 2014) so they are conserved across distant bivalve taxa, and the GO terms associated 404 with such motifs are related to transcriptional control (Supplementary Table 4). An interesting 405 feature of $R$. decussatus LUR is its variable length (Table 6), most likely due to different repeat 406 content. As a matter of fact, the very same repeat sequence was present in every LUR, and our 407 data strongly suggest that LUR length variation is actually due to repeat CNV (see 408 supplementary files on figshare: https://ndownloader.figshare.com/files/8387666 and 409 https://ndownloader.figshare.com/files/8360789), as observed in other bivalve species (see for 410 example Ghiselli et al., 2013; Guerra, Ghiselli \& Passamonti, 2014). Tandem repeats have been 411 also reported in the mitochondrial genomes of the bivalves Acanthocardia tuberculata (Dreyer \& 412 Steiner, 2006), Placopecten magellanicus (La Roche et al., 1990), Moerella iridescens, 413 Sanguinolaria olivacea, Semele scaba, Sinonovacula constricta, Solecurtus divaricatus (Yuan et 414 al., 2012), Ruditapes philippinarum (Ghiselli et al., 2013), and Musculista senhousia (Guerra, 415 Ghiselli \& Passamonti, 2014). These repeats are believed to arise from duplications caused by 416 replication slippage (Buroker et al., 1990; Hayasaka, Ishida \& Horai, 1991; Broughton \& 417 Dowling, 1994). The tandem repeats found at the 3' end of $R$. decussatus LUR are predicted to 418 form a secondary structure (see Figure 2B, and supplementary files on figshare) composed by 419 multiple stem-loops, which obviously increase in number with the increment of the number of 420 tandem repeats. The effect, if any, of tandem repeats in mtDNA is unknown: since the repeats 421 are almost always localized in proximity of the CR, they might interact with regulatory 422 elements - or even contain some - influencing replication and/or transcription initiation, and 423 such interactions might also be altered by the formation of secondary structures (Passamonti et 424 al., 2011; Ghiselli et al., 2013; Guerra, Ghiselli \& Passamonti, 2014). 
425 We assessed the genetic variability of $R$. decussatus mtDNA using two different approaches: by

426 SNP calling in CDS (RNA-Seq data on 12 individuals), and by analysis of the LUR (Sanger

427 sequencing of 14 individuals). The CR and its flanking regions are known to be hypervariable, so

428 they are commonly used to assess polymorphism at low taxonomic levels. Our data strongly

429 support a very low genetic variability: the number of SNPs in CDS is 145, of which 103 are

430 private of a single individual (mRDI01) — thus reducing the number to 42 — while the number of

431 variable sites in the analyzed LURs is 98 over 3,095 aligned positions. Considering the known

432 variability of mtDNA in bivalves (Gissi, Iannelli \& Pesole, 2008; Ghiselli et al., 2013; Breton et

433 al., 2014; Plazzi, Puccio \& Passamonti, 2016), this is a surprising result. Even more if we

434 compare the results of the present work to a methodologically identical analysis performed on 12

435 R. philippinarum samples from the Pacific coast of USA, performed by Ghiselli et al. (2013): in

436 that work, GATK yielded 194 SNPs in the M-type mtDNA and 293 in the F-type. Strikingly, the

43712 R. philippinarum samples analyzed were actually two families (6 siblings +6 siblings). This

438 means that randomly sampled individuals of $R$. decussatus used in this work showed a much

439 lower mtDNA variability than $R$. philippinarum siblings. A previous analysis on the coxl gene of

$440 R$. decussatus reported a nucleotide diversity $(\pi)$ of 0.15 for a population from the Northern

441 Adriatic Sea (Cordero, Peña \& Saavedra, 2014). Another analysis on the same gene of $R$.

442 philippinarum from the same range resulted in a $\pi=0.25$ (Cordero et al., 2017), so $R$. decussatus

443 has a lower nucleotide diversity at the $\operatorname{cox} 1$ locus. The difference between the variability in

444 mtDNA of $R$. decussatus that we are reporting here and that of $R$. philippinarum reported in

445 Ghiselli et al. (2013) appears to be more marked. It is known that the genetic variability of $R$.

446 philippinarum in the Adriatic Sea is lower than in populations from its native range in Asia

447 (Cordero et al., 2017), probably because of the bottlenecks that this species had to go through 
448 during the multiple colonization events. The introduction in North America from Asia happened 449 first (in the 1930s), and from there the Manila clam was introduced in Atlantic Europe (in the 4501970 s and 1980s), and lastly into the Adriatic Sea (1983 and 1984), and it is plausible that the 451 genetic diversity decreased at each introduction event. Accordingly, Cordero et al. (2017) 452 observed that $R$. philippinarum genetic variability in Europe is lower compared to that of the 453 Pacific coast of the USA, so the samples analyzed in Ghiselli et al. (2013) could have been more 454 polymorphic than those analyzed in Cordero et al. (2014), thus explaining the more pronounced 455 differences in genetic variability between the Manila clam and the European clam discussed 456 above. In any case, all the available data point to a lower genetic diversity of $R$. decussatus 457 mtDNA, and it would be interesting to know whether it is a cause or an effect of the ongoing 458 replacement of $R$. decussatus with the invasive $R$. philippinarum. It will also be important to 459 investigate genetic variability of the nuclear genes, especially after Cordero et al. (2014) reported 460 contrasting levels of differentiation between mitochondrial and nuclear markers.

461 With respect to SNP effects, we found 6 indels in CDS, 2 of which do not cause frameshift, but a 462 simple insertion/deletion of 1 amino acid (SNP_1698, and SNP_17619, see Table 8). Of the 463 remaining 4, SNP_6364 and SNP_10449 consist of a deletion and an insertion of a single $\mathrm{T}$ in 464 two homopolymeric sequences (CTTTTTTT and CTTTTTT, respectively), raising the possibility 465 of a sequencing error. In any case, the two SNPs yield a shorter CDS (cytb and $n d 5$, 466 respectively), and are present at relatively low frequencies in the specimens carrying them, 467 except for SNP_6364 which has a frequency of 80\% in fRDI04. The cox3 gene shows 3 SNPs: 468 the first one, SNP_17619, does not cause a frameshift, and results in the deletion of 1 alanine 469 residue, and its frequency in mRDI01 is 97\%. The second one, SNP_17621, consists of a 470 deletion of a $\mathrm{G}$ with respect to the reference sequence, which is the Sanger-sequenced mtDNA of 
471 sample F4; all the individuals analyzed with RNA-Seq carry this deletion except for mRDI01

472 which, at that position, has the same sequence of the reference mtDNA (reference-like allele

473 frequency in $\mathrm{mRDI} 01=99 \%$ ). The third indel, SNP_17624, consists of an insertion of two

474 nucleotides, and its frequency in mRDI01 is 99\%. So, basically, for cox 3 we have three types of

475 sequences: $i$ ) the Sanger-sequenced reference, which yields a 966 bp (321 aa) ORF; ii) a

476 sequence found in 11/12 of samples analyzed with RNA-Seq (except mRDI01) that carries a

477 single-nucleotide deletion (SNP_17621), and yields a 963 bp (320 aa) ORF; iii) a sequence,

478 private of mRDI01, which is obtained by combining SNP_17624 and SNP_17621 (both 99\% of

479 frequency, so most likely co-occurring), which produces a $963 \mathrm{bp}$ (320 aa) ORF. Interestingly,

480 the ORFs obtained from the sequences described in ii) and iii), are almost identical, namely the

481 sequence obtained by RNA-seq in 11/12 samples and the sequence obtained by RNA-Seq in

482 mRDI01 are basically the same, and differ from the Sanger-sequenced reference, yielding an

483 amino acid sequence that differs in the last 35 residues (all data available in supplementary files

484 on figshare: https://doi.org/10.6084/m9.figshare.4970762.v3). Given this consistent difference

485 between the sequence obtained by Sanger-sequencing of DNA, and those obtained by RNA-Seq,

486 it is tempting to speculate that this difference might be caused by RNA editing, a mechanism

487 observed in mtDNA of some animals (Lavrov \& Pett, 2016), and recently reported to be

488 common in cephalopods (Liscovitch-Brauer et al., 2017). Actually, Liscovitch-Brauer et al.

489 (2017) reported only A-to-I editing, which is not the kind of change we are observing here, but

490 other types of editing are known across eukaryotes (see Gott \& Emeson, 2000 for a review), and

491 some others, still unknown, might exist as well. Post-transcriptional modifications (thus

492 including RNA-editing) are still poorly understood mechanisms, but they appear to be

493 responsible for most of the mitochondrial gene expression regulation (Scheibye-Alsing et al., 
494 2007; Scheffler, 2008; Milani et al., 2014a). What we propose here is a pure conjecture, but we 495 think in the future it might be worthy to investigate mitochondrial transcriptomes looking for 496 such kind of "unexpected" biological features.

497 Interestingly, in contrast with a low nucleotide variability along the entire mitochondrial genome, 498 we observed a pretty high polymorphism in LUR length due to CNV of tandem repeats, and even 499 a LUR length heteroplasmy: two females yielded two electrophoretic bands each $(\sim 2,100$ and $500 \sim 3,500$ bp in F3; 2,500 and 3,500 bp in F17; see Table 6). A possible explanation is that the 501 diversity (CNV) detected in the LURs could be recent: the accumulation of nucleotide variation 502 at different sites along the mitochondrial genome needs time, while the kind structural variability 503 we observed can be achieved in few generations (or even one) considering that replication 504 slippage is common in repeat-rich regions.

505

506

Phylogenetic Relationship with R. philippinarum

507 Despite $R$. decussatus and R. philippinarum being morphologically similar and being ascribed to

508 the same genus, the results here reported clearly show that they are quite different both for 509 mtDNA sequence (Figures 3 and 4) and mtDNA gene arrangement (Figure 5). This is an unusual 510 finding, even among bivalves, which are known to be fast-evolving for these characters. This 511 may point to the fact that these two species are less related than previously thought. Actually, 512 this is not the first clue that $R$. decussatus and $R$. philippinarum are quite different genetically, as 513 allozyme electrophoresis (Passamonti, Mantovani \& Scali, 1997, 1999) and satellite DNA 514 content (Passamonti, Mantovani \& Scali, 1998) pointed out. More in-depth analyses are 515 therefore needed to correctly trace the phylogenetic relationships of these two Ruditapes species, 
516 which may eventually end up in two different Genera. As shown in Figures 3, 4 and 5, the Genus

517 Paphia is the most similar to $R$. decussatus.

518

519 Presence/absence of DUI

520 We could not find evidence for sex-specific mtDNAs, typical of DUI. As stated in the

521 Introduction, the search for DUI is not a straightforward process. HTS can help thanks to a much 522 deeper sequencing coverage (in respect to the cloning-and-Sanger-sequencing approach), and

523 because it overcomes the problem of primer specificity, a limitation of the classical approach.

524 One possible concern about using HTS approaches based on short reads in presence of DUI is 525 about the ability of softwares to detect divergent reads and assembly them correctly. More 526 specifically, one could ask what is the divergence threshold under which the assemblers are not 527 able to partition the contigs into two sex-linked groups. We do not know such threshold, but we 528 tried different assembly strategies trying to retrieve sex-specific mtDNA sequences from our 529 data. Other than the approach reported in Materials and Methods (which is the one that produced 530 the data reported here), we tried other techniques. After identifying reads that blasted to bivalve 531 mitochondrial sequences present in GenBank and discarding all the other reads, we generated 532 A5+CAP3 assemblies: $i$ ) for each of the samples (obtaining 12 separate assemblies), and $i i$ ) 533 pooling the 6 males together and the 6 females together, and assembling the two sex-specific 534 pools. Both these approaches did not show evidence of sex-specific mtDNAs. Then we took the 535 assembly obtained from the females and removed the reads from each of the samples that 536 mapped (<8 mismatches) to these sequences. We then used the remaining reads as A5 input. The 537 program could not assemble anything. Lastly, we tried the software MetaVelvet (Namiki et al. 538 2012) — that assembles metagenomes — on all the reads matching bivalve mtDNAs, and only one 
539 genome was produced. After all these alternative approaches failed to find two sex-linked

540 mtDNAs, we decided to proceed with the assembly as indicated in Materials and Methods,

541 because it was the technique that yielded the best quality contigs, most likely because using the

542 reads from all 12 the individuals granted a higher coverage of the mtDNA. Given these results,

543 we can propose three different explanations.

544 1) R. decussatus is characterized by strictly maternal inheritance of mitochondria, so a male-

545 transmitted mtDNA is not present in this species.

546 2) The divergence between the two sex-specific mtDNAs is too low to be detected. This

$547 \quad$ could be the outcome of two different situations.

548

549

550

551

552

553

554

555

556

557

558

559

560

561 a. DUI is very young in this species, so the two sex-linked mtDNAs did not have the time to diverge.

b. A role-reversal event occurred recently. Role reversal (a.k.a. "route reversal" or "masculinization") is a process-observed so far only in species of the Mytilus complex - by which F-type genomes invades the male germ line becoming sperm-transmitted, thus turning into M-type mtDNAs (Hoeh et al. 1997). This event actually resets to zero the divergence between F- and M-type, although substantial differences in the control regions were reported between the original F-type and the "masculinized" one (see Zouros 2013 for a thorough review). The hypothesis that role reversal could have occurred multiple times in the evolutionary history of bivalves and could have led to the complete replacement of M or F mtDNAs in several species was proposed by Hoeh et al. (1996) to explain the scattered phylogenetic distribution of DUI across Bivalvia. Indeed, according to the hypothesis of a single origin, DUI arose $>400$ Mya, 
562

563

564

565

566

567

568

569

570

571

572

573

574

575

576

577

578

579

580

581

582

583 approximately at the origin of Autolamellibranchia, but, as said, such hypothesis requires the assumption of multiple role-reversal and/or DUI loss events in several branches of the bivalve tree (see Zouros, 2013 for a detailed discussion). Recently, a multiple origin of DUI was proposed (Milani et al., 2013; Milani et al., 2014b; Milani, Ghiselli \& Passamonti, 2016; Mitchell et al., 2016), and in such case there would be no need of multiple role-reversal events to explain its phylogeny. In our opinion, until further evidence will be provided, role-reversal should not be considered a rule, but rather an exception. Of course, we cannot rule out that a masculinization event might have occurred in $R$. decussatus, so this hypothesis must be taken into consideration.

3) In our data, even if there is no clear evidence of a male-specific mtDNA, a male sample (mRDI01) clearly stood out from the others, both males and females (see Table 7). Overall, the divergence between mRDI01 and the other 11 samples calculated considering its private SPs is of 151 sites over 18,995 bp (considering the whole mtDNA), and of 103 sites over 14,920 bp (considering only CDS). In both cases the divergence is very low $(0.8 \%$ and $0.7 \%$, respectively), which explains why the mtDNA of mRDI01, although different, was not assembled as a separate genome. We have no sufficient data to evaluate if such divergence is normal within $R$. decussatus populations, but considered the variability usually observed in bivalves, we find the difference unsurprising. On the contrary, the lack of variability among the other 11 samples is remarkable. For these reasons, we are inclined to believe that mRDI01 divergence is compatible with hypotheses 1) and 2). That said, there still could be a third, quite 
584 conjectural, hypothesis by which these data might indicate an incipient DUI, not yet fixed 585 in the population.

586 All in all, we have a preference for the first explanation, but the present data are not sufficient to 587 exclude the others, and a more thorough investigation is necessary to assess this point.

588 Up to now DUI was identified in only 3 Veneridae species: Cyclina sinensis, $R$. philippinarum, 589 and Meretrix lamarckii (Gusman et al., 2016). The status of Paphia is still unknown, and in 590 future works it would be interesting to investigate more Heterodonta species to understand better 591 the distribution of DUI in this derived group of bivalves.

592

593 Acknowledgements

594 We would like to thank Edoardo Turolla (Istituto Delta Ecologia Applicata, Ferrara, Italy) for 595 providing the specimens, and Massimo Milan for bibliographic suggestions. We also gratefully 596 thank the Editor Tim Collins, and the reviewers Carlos Saavedra, Shallee Page, and one 597 anonymous colleague for their comments and suggestions. 


\section{References}

599 Alikhan NF., Petty NK., Ben Zakour NL., Beatson SA. 2011. BLAST Ring Image Generator

600 (BRIG): simple prokaryote genome comparisons. BMC genomics 12:402.

601 Arias-Pérez A., Cordero D., Borrell Y., Sánchez JA., Blanco G., Freire R., Insua A.,

602 Saavedra C. 2016. Assessing the geographic scale of genetic population management with

603 microsatellites and introns in the clam Ruditapes decussatus. Ecology and Evolution 15:3380$604 \quad 3404$.

605 Attwood TK., Bradley P., Flower DR., Gaulton A., Maudling N., Mitchell AL., Moulton G.,

606 Nordle A., Paine K., Taylor P., Uddin A., Zygouri C. 2003. PRINTS and its automatic 607 supplement, prePRINTS. Nucleic acids research 31:400-402.

608 Bailey TL., Boden M., Buske FA., Frith M., Grant CE., Clementi L., Ren J., Li WW., Noble WS. 609 2009. MEME SUITE: tools for motif discovery and searching. Nucleic acids research $610 \quad 37:$ W202-8.

611 Bernt M., Donath A., Jühling F., Externbrink F., Florentz C., Fritzsch G., Pütz J., Middendorf

612 M., Stadler PF. 2013. MITOS: improved de novo metazoan mitochondrial genome 613 annotation. Molecular phylogenetics and evolution 69:313-319.

614 Breton S., Milani L., Ghiselli F., Guerra D., Stewart DT., Passamonti M. 2014. A resourceful 615 genome: updating the functional repertoire and evolutionary role of animal mitochondrial 616 DNAs. Trends in genetics: TIG 30:555-564.

617 Breton S., Stewart DT., Hoeh WR. 2010. Characterization of a mitochondrial ORF from the 618 gender-associated mtDNAs of Mytilus spp. (Bivalvia: Mytilidae): identification of the 619 “missing” ATPase 8 gene. Marine genomics 3:11-18.

620 Breton S., Stewart DT., Shepardson S., Trdan RJ., Bogan AE., Chapman EG., Ruminas AJ., 
621 Piontkivska H., Hoeh WR. 2011. Novel protein genes in animal mtDNA: a new sex

622 determination system in freshwater mussels (Bivalvia: Unionoida)? Molecular biology and 623 evolution 28:1645-1659.

624 Broughton RE., Dowling TE. 1994. Length variation in mitochondrial DNA of the minnow 625 Cyprinella spiloptera. Genetics 138:179-190.

626 Buroker NE., Brown JR., Gilbert TA., O’Hara PJ., Beckenbach AT., Thomas WK., Smith MJ.

627 1990. Length heteroplasmy of sturgeon mitochondrial DNA: an illegitimate elongation 628 model. Genetics 124:157-163.

629 Buske FA., Bodén M., Bauer DC., Bailey TL. 2010. Assigning roles to DNA regulatory motifs 630 using comparative genomics. Bioinformatics 26:860-866.

631 Cao L., Kenchington E., Zouros E., Rodakis GC. 2004. Evidence that the large noncoding 632 sequence is the main control region of maternally and paternally transmitted mitochondrial 633 genomes of the marine mussel (Mytilus spp.). Genetics 167:835-850.

634 Capella-Gutiérrez S., Silla-Martinez JM., Gabaldón T. 2009. trimAl: a tool for automated 635 alignment trimming in large-scale phylogenetic analyses. Bioinformatics 25:1972-1973. 636 Chamary JV., Parmley JL., Hurst LD. 2006. Hearing silence: non-neutral evolution at 637 synonymous sites in mammals. Nature reviews. Genetics 7:98-108.

638 Cordero D., Delgado M., Liu B., Ruesink J., Saavedra C. 2017. Population genetics of the 639 Manila clam (Ruditapes philippinarum) introduced in North America and Europe. Scientific $640 \quad$ reports $7: 39745$.

641 Cordero D., Peña JB., Saavedra C. 2014. Phylogeographic analysis of introns and mitochondrial 642 DNA in the clam Ruditapes decussatus uncovers the effects of Pleistocene glaciations and 643 endogenous barriers to gene flow. Molecular phylogenetics and evolution 71:274-287. 
644 Crick FHC. 1966. Codon — anticodon pairing: The wobble hypothesis. Journal of molecular 645 biology 19:548-555.

646 Darty K., Denise A., Ponty Y. 2009. VARNA: Interactive drawing and editing of the RNA 647 secondary structure. Bioinformatics 25:1974-1975.

648 DePristo MA., Banks E., Poplin R., Garimella KV., Maguire JR., Hartl C., Philippakis AA., del 649 Angel G., Rivas MA., Hanna M., McKenna A., Fennell TJ., Kernytsky AM., Sivachenko 650 AY., Cibulskis K., Gabriel SB., Altshuler D., Daly MJ. 2011. A framework for variation 651

652 discovery and genotyping using next-generation DNA sequencing data. Nature genetics $43: 491-498$

653

654

655

656

657

658

659

660

661

662

663

664

665 666

Diz AP., Dudley E., Skibinski DOF. 2012. Identification and characterisation of highly expressed proteins in sperm cells of the marine mussel Mytilus edulis. Proteomics. DOI: 10.1002/pmic.201100500.

Dreyer H., Steiner G. 2006. The complete sequences and gene organisation of the mitochondrial genomes of the heterodont bivalves Acanthocardia tuberculata and Hiatella arctica-and

the first record for a putative Atpase subunit 8 gene in marine bivalves. Frontiers in zoology 3:13.

Finn RD., Coggill P., Eberhardt RY., Eddy SR., Mistry J., Mitchell AL., Potter SC., Punta M., Qureshi M., Sangrador-Vegas A., Salazar GA., Tate J., Bateman A. 2016. The Pfam protein families database: towards a more sustainable future. Nucleic acids research 44:D279-85.

Ghiselli F., Milani L., Chang PL., Hedgecock D., Davis JP., Nuzhdin SV., Passamonti M. 2012. De Novo assembly of the Manila clam Ruditapes philippinarum transcriptome provides new insights into expression bias, mitochondrial doubly uniparental inheritance and sex determination. Molecular biology and evolution 29:771-786. 
667 Ghiselli F., Milani L., Guerra D., Chang PL., Breton S., Nuzhdin SV., Passamonti M. 2013.

668 Structure, transcription, and variability of metazoan mitochondrial genome: perspectives

669 from an unusual mitochondrial inheritance system. Genome biology and evolution 5:1535-

$670 \quad 1554$.

671 Ghiselli F., Milani L., Passamonti M. 2011. Strict sex-specific mtDNA segregation in the germ

672 line of the DUI species Venerupis philippinarum (Bivalvia: Veneridae). Molecular biology

673 and evolution 28:949-961.

674 Gissi C., Iannelli F., Pesole G. 2008. Evolution of the mitochondrial genome of Metazoa as

675 exemplified by comparison of congeneric species. Heredity 101:301-320.

676 Gosling EM. 2003. Bivalve molluscs. Wiley Online Library.

677 Gott JM., Emeson RB. 2000. Functions and mechanisms of RNA editing. Annual Review of $678 \quad$ Genetics. 34:499-531.

679 Gruber AR., Lorenz R., Bernhart SH., Neuböck R., Hofacker IL. 2008. The Vienna RNA

$680 \quad$ websuite. Nucleic acids research 36:W70-4.

681 Guerra D., Ghiselli F., Passamonti M. 2014. The largest unassigned regions of the male- and 682 female-transmitted mitochondrial DNAs in Musculista senhousia (Bivalvia Mytilidae).

$683 \quad$ Gene 536:316-325.

684 Gusman A., Lecomte S., Stewart DT., Passamonti M., Breton S. 2016. Pursuing the quest for

685 better understanding the taxonomic distribution of the system of doubly uniparental 686 inheritance of mtDNA. PeerJ 4:e2760.

687 Hayasaka K., Ishida T., Horai S. 1991. Heteroplasmy and polymorphism in the major noncoding 688 region of mitochondrial DNA in Japanese monkeys: association with tandemly repeated 689 sequences. Molecular biology and evolution 8:399-415. 
690 He Z., Zhang H., Gao S., Lercher MJ., Chen W-H., Hu S. 2016. Evolview v2: an online 691 visualization and management tool for customized and annotated phylogenetic trees. $692 \quad$ Nucleic acids research 44:W236-41.

693 Hoeh WR., Stewart DT., Saavedra C., Sutherland BW., Zouros E. 1997. Phylogenetic evidence 694 for role-reversals of gender-associated mitochondrial DNA in Mytilus (Bivalvia: Mytilidae). $695 \quad$ Molecular Biology and Evolution 14:959-967.

696 Huang X., Madan A. 1999. CAP3: A DNA sequence assembly program. Genome research $697 \quad 9: 868-877$.

698 Jones P., Binns D., Chang H-YY., Fraser M., Li W., McAnulla C., McWilliam H., Maslen J., 699 Mitchell A., Nuka G., et al. 2014. InterProScan 5: genome-scale protein function $700 \quad$ classification. Bioinformatics 30:1236-1240.

701 Ju YS., Kim J-I., Kim S., Hong D., Park H., Shin J-Y., Lee S., Lee W-C., Kim S., Yu S-B., et al. 702 2011. Extensive genomic and transcriptional diversity identified through massively parallel 703 DNA and RNA sequencing of eighteen Korean individuals. Nature Genetics. 43:745-752.

704 King JL., LaRue BL., Novroski NM., Stoljarova M., Seo SB., Zeng X., Warshauer DH., Davis 705 CP., Parson W., Sajantila A., et al. 2014. High-quality and high-throughput massively 706 parallel sequencing of the human mitochondrial genome using the Illumina MiSeq. $707 \quad$ Forensic Science International: Genetics. 12:128-135.

708 La Roche J., Snyder M., Cook DI., Fuller K., Zouros E. 1990. Molecular characterization of a 709 repeat element causing large-scale size variation in the mitochondrial DNA of the sea 710 scallop Placopecten magellanicus. Molecular biology and evolution 7:45-64.

711 Laslett D., Canback B. 2008. ARWEN: a program to detect tRNA genes in metazoan 712 mitochondrial nucleotide sequences. Bioinformatics 24:172-175. 
713 Lavrov DV., Pett W. 2016. Animal Mitochondrial DNA as We Do Not Know It: mt-Genome

714 Organization and Evolution in Nonbilaterian Lineages. Genome biology and evolution

$715 \quad 8: 2896-2913$.

716 Leite RB., Milan M., Coppe A., Bortoluzzi S., dos Anjos A., Reinhardt R., Saavedra C.,

717 Patarnello T., Cancela ML., Bargelloni L. 2013. mRNA-Seq and microarray development

718 for the Grooved Carpet shell clam, Ruditapes decussatus: a functional approach to unravel

719 host-parasite interaction. BMC genomics 14:741.

720 Liscovitch-Brauer N., Alon S., Porath HT., Elstein B., Unger R., Ziv T., Admon A., Levanon

721 EY., Rosenthal JJC., Eisenberg E. 2017. Trade-off between Transcriptome Plasticity and

722 Genome Evolution in Cephalopods. Cell 169:191-202.e11.

723 Lubośny M., Przyłucka A., Sańko TJ., Śmietanka B., Rosenfeld S., Burzyński A. 2017/2. Next

724 generation sequencing of gonadal transcriptome suggests standard maternal inheritance of

725 mitochondrial DNA in Eurhomalea rufa (Veneridae). Marine genomics 31:21-23.

726 McKenna A., Hanna M., Banks E., Sivachenko A., Cibulskis K., Kernytsky A., Garimella K.,

727 Altshuler D., Gabriel S., Daly M., DePristo MA. 2010. The Genome Analysis Toolkit: a

728 MapReduce framework for analyzing next-generation DNA sequencing data. Genome

729 research 20:1297-1303.

730 Milani L., Ghiselli F. 2015. Mitochondrial activity in gametes and transmission of viable

731 mtDNA. Biology direct 10:22.

732 Milani L., Ghiselli F., Guerra D., Breton S., Passamonti M. 2013. A comparative analysis of

733 mitochondrial ORFans: new clues on their origin and role in species with doubly

734 uniparental inheritance of mitochondria. Genome biology and evolution 5:1408-1434.

735 Milani L., Ghiselli F., Iannello M., Passamonti M. 2014a. Evidence for somatic transcription of 
male-transmitted mitochondrial genome in the DUI species Ruditapes philippinarum

737 (Bivalvia: Veneridae). Current genetics 60:163-173.

738

739

740

741

742

743

744

745

746

747

748

749

750

751

752

753

754

755

756

757

758

Milani L., Ghiselli F., Maurizii MG., Nuzhdin SV., Passamonti M. 2014b. Paternally transmitted mitochondria express a new gene of potential viral origin. Genome biology and evolution 6:391-405.

Milani L., Ghiselli F., Passamonti M. 2016. Mitochondrial selfish elements and the evolution of biological novelties. Current zoology 62:687-697.

Milbury CA., Lee JC., Cannone JJ., Gaffney PM., Gutell RR. 2010. Fragmentation of the large subunit ribosomal RNA gene in oyster mitochondrial genomes. BMC genomics 11:485.

Mitchell A., Guerra D., Stewart D., Breton S. 2016. In silico analyses of mitochondrial ORFans in freshwater mussels (Bivalvia: Unionoida) provide a framework for future studies of their origin and function. BMC Genomics 17:597.

Mortazavi A., Williams BA., McCue K., Schaeffer L., Wold B. 2008. Mapping and quantifying mammalian transcriptomes by RNA-Seq. Nature methods 5:621-628.

Namiki T., Hachiya T., Tanaka H., Sakakibara Y. 2012. MetaVelvet: an extension of

Velvet assembler to de novo metagenome assembly from short sequence reads.

Nucleic Acids Research. 40:e155.

Ojala D., Montoya J., Attardi G. 1981. tRNA punctuation model of RNA processing in human mitochondria. Nature. 290:470-4.

Passamonti M., Ghiselli F. 2009. Doubly uniparental inheritance: two mitochondrial genomes, one precious model for organelle DNA inheritance and evolution. DNA and cell biology 28:79-89.

Passamonti M., Mantovani B., Scali V. 1997. Allozymic characterization and genetic 

zoology 64:117-124.

761

Passamonti M., Mantovani B., Scali V. 1998. Characterization of a highly repeated DNA family in tapetinae species (mollusca bivalvia: veneridae). Zoological science 15:599-605.

Passamonti M., Mantovani B., Scali V. 1999. Allozymic analysis of some Mediterranean Veneridae (Mollusca: Bivalvia): preliminary notes on taxonomy and systematics of the family. Journal of the Marine Biological Association of the United Kingdom. Marine Biological Association of the United Kingdom 79:899-906.

Passamonti M., Ricci A., Milani L., Ghiselli F. 2011. Mitochondrial genomes and Doubly Uniparental Inheritance: new insights from Musculista senhousia sex-linked mitochondrial DNAs (Bivalvia Mytilidae). BMC genomics 12:442.

Pesole G., Allen JF., Lane N., Martin W., Rand DM., Schatz G., Saccone C. 2012. The neglected genome. EMBO reports 13:473-474.

Plazzi F., Puccio G., Passamonti M. 2016. Comparative Large-Scale Mitogenomics Evidences Clade-Specific Evolutionary Trends in Mitochondrial DNAs of Bivalvia. Genome biology and evolution 8:2544-2564.

Pozzi A., Plazzi F., Milani L., Ghiselli F., Passamonti M. 2017. SmithRNAs: could mitochondria "bend" nuclear regulation? Molecular biology and evolution. DOI: 10.1093/molbev/msx140.

Rozen S., Skaletsky H. 2000. Primer3 on the WWW for general users and for biologist programmers. Methods in molecular biology 132:365-386.

Scheffler IE. 2008. Mitochondria. Hoboken, N.J.: Wiley.

Scheibye-Alsing K., Cirera S., Gilchrist MJ., Fredholm M., Gorodkin J. 2007. EST analysis on 
782

783

784 Serb JM., Lydeard C. 2003. Complete mtDNA sequence of the North American freshwater 785

786

787

788

789

790

791

792

793

794

795

796

797

798

799

800

801

802

803

804

pig mitochondria reveal novel expression differences between developmental and adult tissues. BMC genomics 8:367. mussel, Lampsilis ornata (Unionidae): an examination of the evolution and phylogenetic utility of mitochondrial genome organization in Bivalvia (Mollusca). Molecular biology and evolution 20:1854-1866.

Sievers F., Wilm A., Dineen D., Gibson TJ., Karplus K., Li W., Lopez R., McWilliam H., Remmert M., Söding J., Thompson JD., Higgins DG. 2011. Fast, scalable generation of high-quality protein multiple sequence alignments using Clustal Omega. Molecular systems biology 7:539.

Skibinski DO., Gallagher C., Beynon CM. 1994a. Mitochondrial DNA inheritance. Nature $368: 817-818$.

Skibinski DO., Gallagher C., Beynon CM. 1994b. Sex-limited mitochondrial DNA transmission in the marine mussel Mytilus edulis. Genetics 138:801-809.

Smith DR. 2013. RNA-Seq data: a goldmine for organelle research. Briefings in functional genomics 12:454-456.

de Sousa JT., Milan M., Bargelloni L., Pauletto M., Matias D., Joaquim S., Matias AM., Quillien V., Leitão A., Huvet A. 2014. A microarray-based analysis of gametogenesis in two Portuguese populations of the European clam Ruditapes decussatus. PloS one 9:e92202.

Stamatakis A. 2014. RAxML version 8: a tool for phylogenetic analysis and post-analysis of large phylogenies. Bioinformatics 30:1312-1313.

Stothard P. 2000. The sequence manipulation suite: JavaScript programs for analyzing and formatting protein and DNA sequences. BioTechniques 28:1102, 1104. 
805 Sullivan MJ., Petty NK., Beatson SA. 2011. Easyfig: a genome comparison visualizer. $806 \quad$ Bioinformatics 27:1009-1010.

807 Tamura K., Stecher G., Peterson D., Filipski A., Kumar S. 2013. MEGA6: Molecular 808 Evolutionary Genetics Analysis version 6.0. Molecular biology and evolution 30:27258092729.

810 Tan MH., Gan HM., Schultz MB., Austin CM. 2015. MitoPhAST, a new automated 811 mitogenomic phylogeny tool in the post-genomic era with a case study of 89 decapod 812 mitogenomes including eight new freshwater crayfish mitogenomes. Molecular 813 phylogenetics and evolution. DOI: 10.1016/j.ympev.2015.02.009.

814 Theologidis I., Fodelianakis S., Gaspar MB., Zouros E. 2008. Doubly uniparental inheritance 815 (DUI) of mitochondrial DNA in Donax trunculus (Bivalvia: Donacidae) and the problem of 816 its sporadic detection in Bivalvia. Evolution; international journal of organic evolution $817 \quad 62: 959-970$.

818 Tritt A., Eisen JA., Facciotti MT., Darling AE. 2012. An integrated pipeline for de novo 819 assembly of microbial genomes. PloS one 7:e42304.

820 Wheeler DL., Church DM., Federhen S., Lash AE., Madden TL., Pontius JU., Schuler GD., 821 Schriml LM., Sequeira E., Tatusova TA., Wagner L. 2005. Database resources of the 822 National Center for Biotechnology. Nucleic Acid Research 33:D39-D45.

823 Yu H., Li Q. 2011. Mutation and selection on the wobble nucleotide in tRNA anticodons in 824 marine bivalve mitochondrial genomes. PLoS One 6:e16147.

825 Yuan Y., Li Q., Yu H., Kong L. 2012. The complete mitochondrial genomes of six heterodont 826 bivalves (Tellinoidea and Solenoidea): variable gene arrangements and phylogenetic 827 implications. PloS one 7:e32353. 
828 Yuan S., Xia Y., Zheng Y., Zeng X. 2016. Next-generation sequencing of mixed genomic DNA 829 allows efficient assembly of rearranged mitochondrial genomes in Amolops chunganensis $830 \quad$ and Quasipaa boulengeri. PeerJ 4:e2786.

831 Zouros E. 2013. Biparental Inheritance Through Uniparental Transmission: The Doubly 832 Uniparental Inheritance (DUI) of Mitochondrial DNA. Evolutionary biology 40:1-31.

833 Zouros E., Ball AO., Saavedra C., Freeman KR. 1994a. Mitochondrial DNA inheritance. Nature $834 \quad 368: 818$

835 Zouros E., Oberhauser Ball A., Saavedra C., Freeman KR. 1994b. An unusual type of 836 mitochondrial DNA inheritance in the blue mussel Mytilus. Proceedings of the National 837 Academy of Sciences of the United States of America 91:7463-7467. 
Figure 1 (on next page)

$R$. decussatus mtDNA gene arrangement 


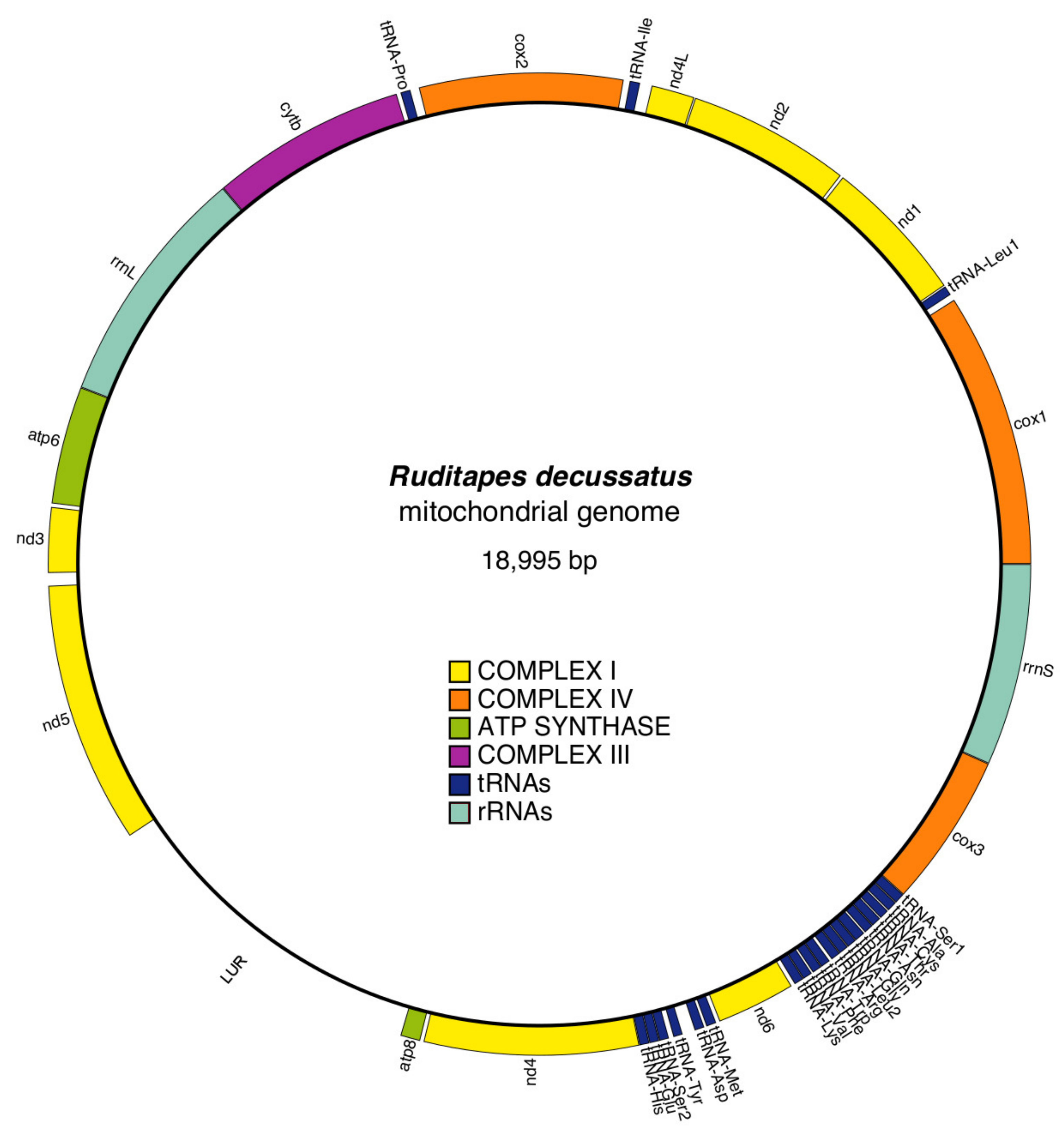


Figure 2 (on next page)

Principal features of the Largest Unassigned Region (LUR)

A: map of the IUR; B: DNA secondary structure predicted in the repeat region (boxed in A); C: Logos of the two DNA motifs found in the LUR. 
A

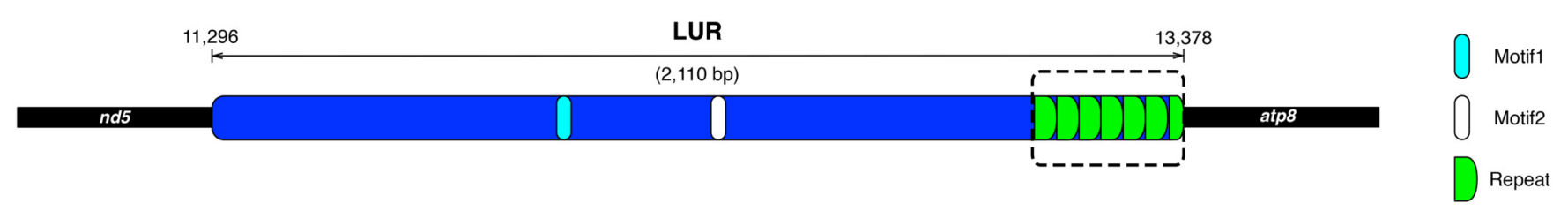

B

C
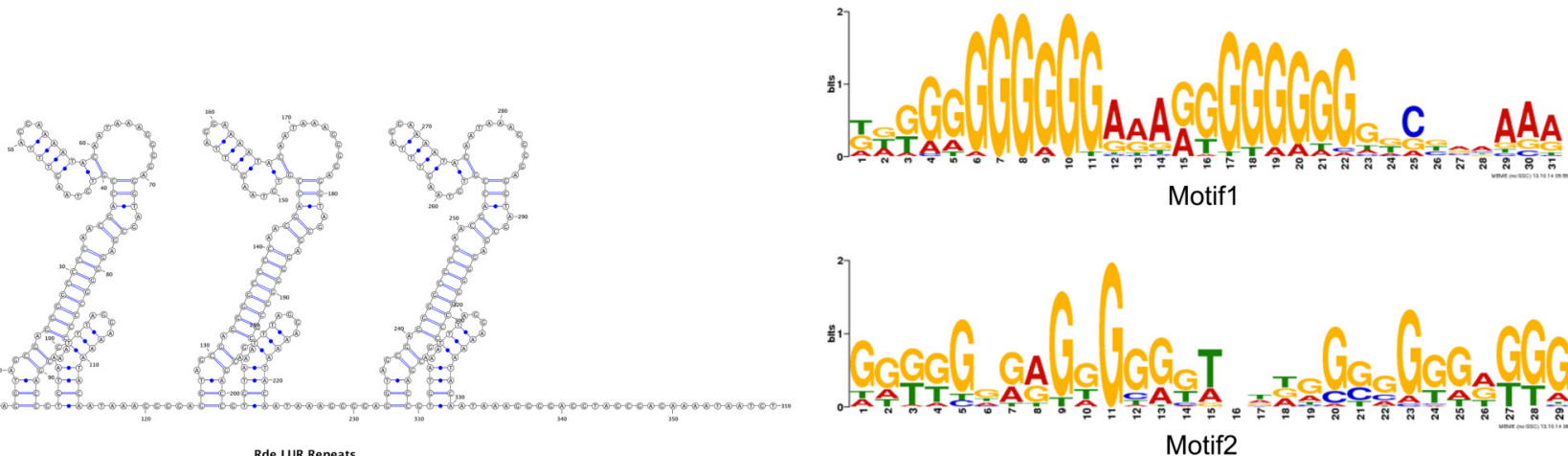

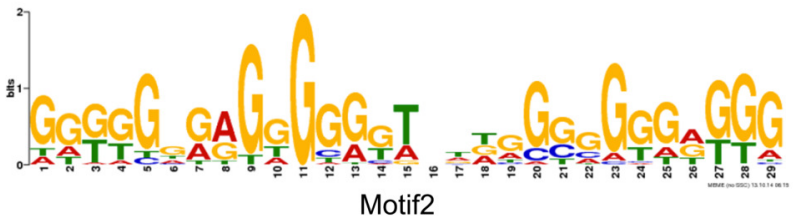


Figure 3 (on next page)

BLASTN comparison of $R$. decussatus and other Veneridae

R. decussatus mtDNA map (external gray circle), and BLASTN identity (colored inner circles) with complete mtDNAs of other 10 venerid species (see list in Supplementary Table 3). 
Manuscript to be reviewed

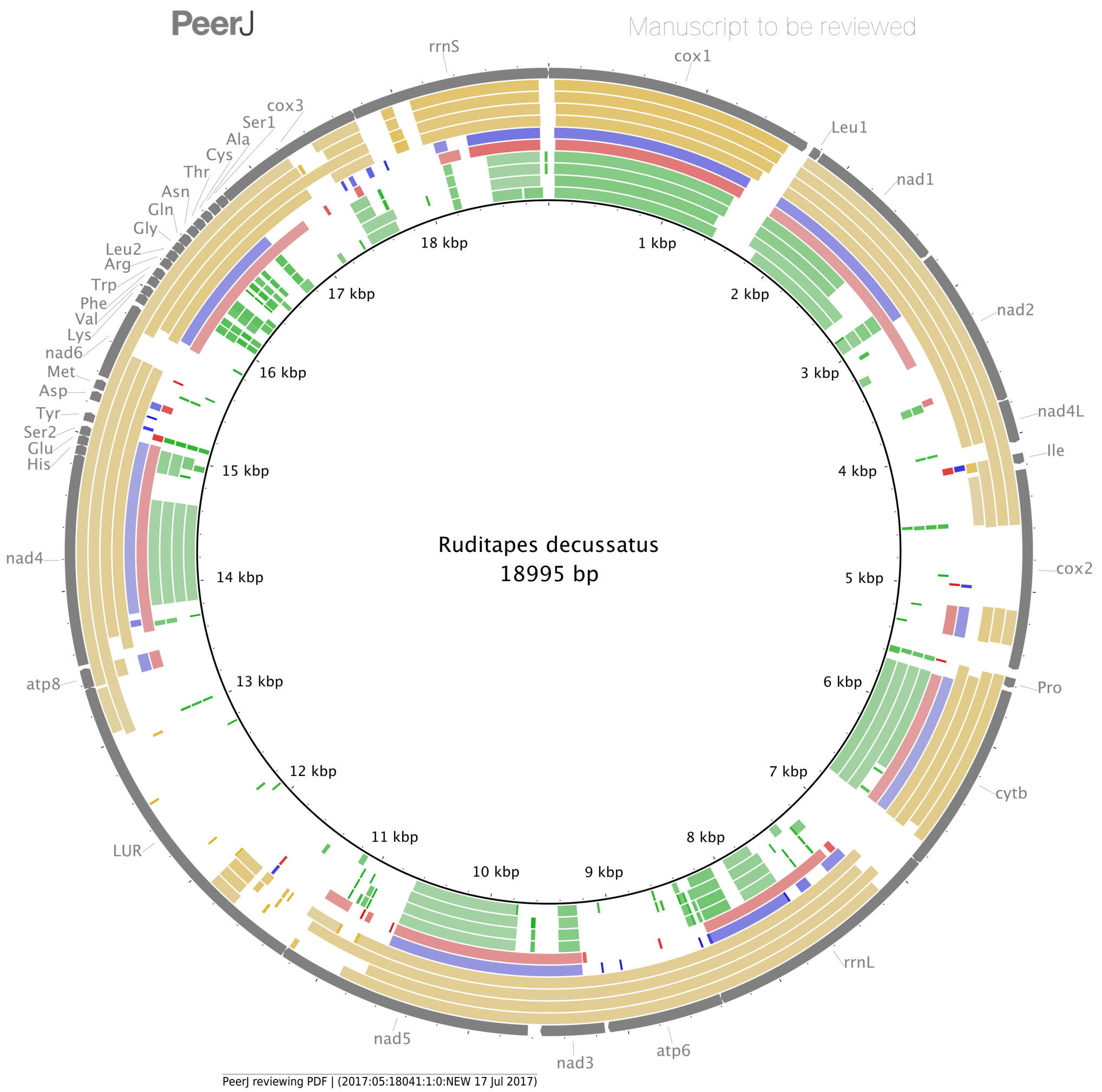

Meretrix lamarckii

$100 \%$ identity

$70 \%$ identity

$50 \%$ identity

Meretrix lusoria

$100 \%$ identity

70\% identity

$50 \%$ identity

Meretrix meretrix

$100 \%$ identity

70\% identity

$50 \%$ identity

Meretrix petechialis

$100 \%$ identity

$70 \%$ identity

50\% identity

Ruditapes philippinarum F-Type

$100 \%$ identity

70\% identity

$50 \%$ identity

Ruditapes philippinarum M-Type

100\% identity

70\% identity

50\% identity

Paphia amabilis

$100 \%$ identity

70\% identity

50\% identity

Paphia euglypta

$100 \%$ identity

$70 \%$ identity

$50 \%$ identity

Paphia textile

$100 \%$ identity

$70 \%$ identity

50\% identity

Paphia undulata

$100 \%$ identity

70\% identity

$50 \%$ identity

Annotation 


\section{Figure 4 (on next page)}

Maximum Likelihood (ML) tree of Veneridae obtained with all mitochondrial coding genes.

ML tree obtained with the MitoPhast pipeline; the complete input and output of this analysis is available on figshare (https://doi.org/10.6084/m9.figshare.4970762.v1). 


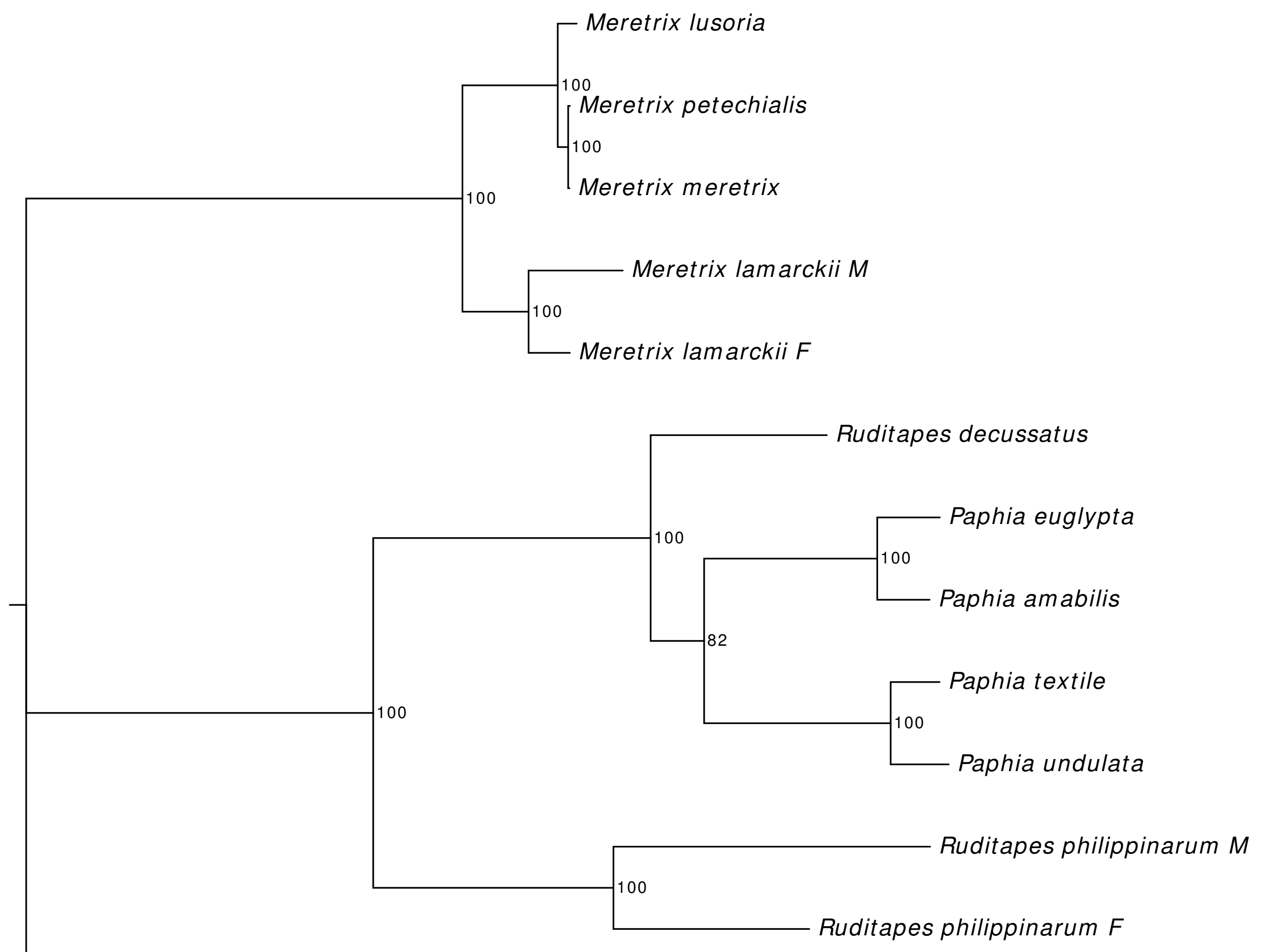

Arctica islandica 
Figure $\mathbf{5}$ (on next page)

Comparison of gene order in venerid mtDNAs

Variation in gene order between $R$. decussatus and P. euglypta (Figure 5A), M. lamarckii Ftype (Figure 5B), $R$. philippinarum F-type (Figure $5 C$ ), and among all the 4 species (Figure $5 D)$. 



\section{Table 1 (on next page)}

Features of the contigs obtained by de novo assembly of mtDNA. 
1 Table 1 Features of the contigs obtained by de novo assembly of mtDNA.

2

\begin{tabular}{|c|c|c|c|c|}
\hline Contig & Length & Gene content & Poly-A & Notes \\
\hline 1 & 6794 & $\begin{array}{l}\text { atp6_nd3_nd5_cox1_tRNA- } \\
\text { Leu1_nd1_nd2_nd4L }\end{array}$ & Yes & $\begin{array}{l}\text { Chimeric assembly. The contiguity } \\
\text { between } n d 5 \text { and } \operatorname{cox} 1 \text { is an artifact }\end{array}$ \\
\hline 2 & 1884 & rrnS_cox3 & No & - \\
\hline 3 & 1288 & atp6_nd3 & Yes & - \\
\hline 4 & 1663 & $\operatorname{cox} 3$ & $?$ & The contig ends with just 8 As \\
\hline 5 & 1934 & $\begin{array}{l}\text { atp8_nd4_tRNA-His_tRNA- } \\
\text { Glu_tRNA-Ser2_tRNA-Tyr }\end{array}$ & No & - \\
\hline 6 & 1831 & atp8_nd4_tRNA-His & Yes & - \\
\hline 7 & 5478 & $\begin{array}{l}\operatorname{cox} 2 \_t R N A- \\
\text { Ile_nd4L_nd2_nd1_tRNA- } \\
\text { Leu1_cox1 }\end{array}$ & Yes & $\begin{array}{l}\text { There is a polyadenylation signal } \\
\text { (56 As) after the cox } 2 \text { gene }\end{array}$ \\
\hline 8 & 2879 & cytb_rrnL & No & - \\
\hline 9 & 952 & $\begin{array}{l}\text { nd6_tRNA-Lys_tRNA- } \\
\text { Val_tRNA-Phe_tRNA- } \\
\text { Trp_tRNA-Arg_tRNA-Leu2 }\end{array}$ & No & - \\
\hline
\end{tabular}




\section{Table 2 (on next page)}

MtDNA gene arrangement of $R$. decussatus.

The anticodon of tRNAs are reported in the $5^{\prime}-3^{\prime}$ direction. 
1 Table 2 MtDNA gene arrangement of $R$. decussatus. The anticodon of tRNAs are 2 reported in the 5'-3' direction.

3

\begin{tabular}{|c|c|c|c|c|c|c|c|}
\hline Name & Type & Start & Stop & Length (bp) & Start & Stop & Anticodon \\
\hline $\operatorname{cox} 1$ & Coding & 1 & 1,716 & 1,716 & ATA & TAG & \\
\hline tRNA-Leu1 & tRNA & 1,754 & 1,815 & 62 & & & TAG \\
\hline$n d 1$ & Coding & 1,822 & 2,739 & 918 & ATA & TAA & \\
\hline nd2 & Coding & 2,755 & 3,774 & 1,020 & ATG & TAG & \\
\hline$n d 4 I$ & Coding & 3,780 & 4,052 & 273 & ATA & TAG & \\
\hline tRNA-IIe & tRNA & 4,125 & 4,190 & 66 & & & GAT \\
\hline $\operatorname{cox} 2$ & Coding & 4,228 & 5,499 & 1,272 & ATA & TAG & \\
\hline tRNA-Pro & tRNA & 5,553 & 5,616 & 64 & & & TGG \\
\hline cytb & Coding & 5,641 & 6,864 & 1,224 & ATA & TAG & \\
\hline$r r n L$ & rRNA & 6,865 & 8,385 & 1,521 & & & \\
\hline atp6 & Coding & 8,386 & 9,123 & 738 & ATG & TAA & \\
\hline$n d 3$ & Coding & 9,145 & 9,552 & 408 & ATG & TAA & \\
\hline$n d 5$ & Coding & 9,631 & 11,268 & 1,638 & ATG & TAG & \\
\hline atp8 & Coding & 13,379 & 13,504 & 126 & ATA & TAA & \\
\hline$n d 4$ & Coding & 13,526 & 14,865 & 1,340 & ATA & $\mathrm{TA}^{*}$ & \\
\hline tRNA-His & tRNA & 14,866 & 14,928 & 63 & & & GTG \\
\hline tRNA-Glu & tRNA & 14,929 & 14,990 & 62 & & & TTC \\
\hline tRNA-Ser2 & tRNA & 14,991 & 15,052 & 62 & & & TGA \\
\hline tRNA-Tyr & tRNA & 15,081 & 15,140 & 60 & & & GTA \\
\hline tRNA-Asp & tRNA & 15,218 & 15,280 & 63 & & & GTC \\
\hline tRNA-Met & tRNA & 15,294 & 15,358 & 65 & & & CAT \\
\hline$n d 6$ & Coding & 15,380 & 15,874 & 495 & ATG & TAA & \\
\hline tRNA-Lys & tRNA & 15,897 & 15,959 & 63 & & & TTT \\
\hline tRNA-Val & tRNA & 15,960 & 16,021 & 62 & & & TAC \\
\hline tRNA-Phe & tRNA & 16,030 & 16,092 & 63 & & & GAA \\
\hline tRNA-Trp & tRNA & 16,093 & 16,155 & 63 & & & TCA \\
\hline tRNA-Arg & tRNA & 16,171 & 16,232 & 62 & & & TCG \\
\hline tRNA-Leu2 & tRNA & 16,233 & 16,295 & 63 & & & TAA \\
\hline tRNA-Gly & tRNA & 16,297 & 16,358 & 62 & & & TCC \\
\hline$t R N A-G / n$ & tRNA & 16,359 & 16,427 & 69 & & & TTG \\
\hline tRNA-Asn & tRNA & 16,435 & 16,497 & 63 & & & GTT \\
\hline tRNA-Thr & tRNA & 16,498 & 16,560 & 63 & & & TGT \\
\hline tRNA-Cys & tRNA & 16,565 & 16,626 & 62 & & & GCA \\
\hline tRNA-Ala & tRNA & 16,632 & 16,696 & 65 & & & TGC \\
\hline tRNA-Ser1 & tRNA & 16,698 & 16,764 & 67 & & & TCT \\
\hline $\operatorname{cox} 3$ & Coding & 16,765 & 17,730 & 966 & ATG & TAA & \\
\hline$r r n S$ & rRNA & 17,731 & 18,995 & 1,265 & & & \\
\hline
\end{tabular}


13

14

15

16

17 
Table $\mathbf{3}$ (on next page)

Unassigned Regions (URs) 
1 Table 3 Unassigned Regions (URs)

2

3

\begin{tabular}{|l|c|c|c|}
\hline \multicolumn{1}{|c|}{ UR name } & Start & Stop & Length (bp) \\
\hline UR1 & 1,717 & 1,753 & 37 \\
\hline UR2 & 1,816 & 1,821 & 6 \\
\hline UR3 & 2,740 & 2,754 & 15 \\
\hline UR4 & 3,775 & 3,779 & 5 \\
\hline UR5 & 4,053 & 4,124 & 72 \\
\hline UR6 & 4,191 & 4,227 & 37 \\
\hline UR7 & 5,500 & 5,552 & 53 \\
\hline UR8 & 5,617 & 5,640 & 24 \\
\hline UR9 & 9,124 & 9,144 & 21 \\
\hline UR10 & 9,553 & 9,630 & 78 \\
\hline UR11 (LUR) & 11,269 & 13,378 & 2,110 \\
\hline UR12 & 13,505 & 13,525 & 21 \\
\hline UR13 & 15,053 & 15,080 & 28 \\
\hline UR14 & 15,141 & 15,217 & 77 \\
\hline UR15 & 15,281 & 15,293 & 13 \\
\hline UR16 & 15,359 & 15,379 & 21 \\
\hline UR17 & 15,875 & 15,896 & 22 \\
\hline UR18 & 16,022 & 16,029 & 8 \\
\hline UR19 & 16,156 & 16,170 & 15 \\
\hline UR20 & 16,296 & 16,296 & 1 \\
\hline UR21 & 16,428 & 16,434 & 7 \\
\hline UR22 & 16,561 & 16,564 & 4 \\
\hline UR23 & 16,627 & 16,631 & 5 \\
\hline UR24 & 16,697 & 16,697 & 1 \\
\hline
\end{tabular}

4

5

6 


\section{Table 4(on next page)}

Nucleotide composition.

URs $=$ Unassigned Regions 
Table 4 Nucleotide composition. URs = Unassigned Regions.

2

\begin{tabular}{|l|c|c|c|c|c|c|c|c|c|c|c|}
\hline \multicolumn{1}{|c|}{ Name } & Length (bp) & T \% & C \% & A \% & G \% & A+T \% & T3 \% & C3 \% & A3 \% & G3 \% & A3+T3 \% \\
\hline cox1 & 1,716 & 35.8 & 15.5 & 25.8 & 22.9 & 61.6 & 39 & 12.1 & 28.0 & 21.3 & 67.0 \\
\hline$n d 1$ & 918 & 38.7 & 12.5 & 24.0 & 24.8 & 62.7 & 38 & 10.1 & 30.7 & 21.2 & 68.7 \\
\hline$n d 2$ & 1,020 & 38.3 & 11.0 & 24.8 & 25.9 & 63.1 & 35 & 11.5 & 29.4 & 24.4 & 64.4 \\
\hline$n d 4 I$ & 273 & 39.9 & 12.8 & 25.3 & 22.0 & 65.2 & 34 & 14.3 & 30.8 & 20.9 & 64.8 \\
\hline cox2 & 1,272 & 29.7 & 14.8 & 29.1 & 26.4 & 58.8 & 30 & 15.3 & 27.4 & 27.6 & 57.4 \\
\hline cob & 1,224 & 37.4 & 17.2 & 22.7 & 22.6 & 60.1 & 41 & 14.7 & 21.8 & 22.1 & 62.8 \\
\hline$r$ rnL & 1,749 & 33.2 & 11.5 & 32.6 & 22.6 & 65.8 & 33 & 10.6 & 33.4 & 23.0 & 66.4 \\
\hline atp6 & 510 & 42.0 & 15.7 & 20.8 & 21.6 & 62.8 & 45 & 13.5 & 21.8 & 20.0 & 66.8 \\
\hline nd3 & 408 & 39.5 & 11.0 & 24.8 & 24.8 & 64.3 & 33 & 11.0 & 30.1 & 25.7 & 63.1 \\
\hline$n d 5$ & 1,638 & 37.6 & 11.7 & 27.7 & 23.0 & 65.3 & 35 & 11.0 & 34.2 & 19.8 & 69.2 \\
\hline atp8 & 126 & 44.4 & 11.9 & 19.0 & 24.6 & 63.4 & 45 & 4.8 & 23.8 & 26.2 & 68.8 \\
\hline$n d 4$ & 1,340 & 38.9 & 12.9 & 22.1 & 26.1 & 61.0 & 41 & 10.8 & 24.9 & 23.5 & 65.9 \\
\hline nd6 & 495 & 39.2 & 12.1 & 23.0 & 25.7 & 62.2 & 38 & 13.9 & 27.9 & 20.0 & 65.9 \\
\hline cox3 & 966 & 36.9 & 12.7 & 24.8 & 25.6 & 61.7 & 39 & 9.6 & 28.6 & 23.0 & 67.6 \\
\hline rrnS & 1,265 & 32.7 & 12.3 & 32.9 & 22.1 & 65.6 & 35 & 13.5 & 31.6 & 19.5 & 66.6 \\
\hline All coding & 14,920 & 36.3 & 13.2 & 26.5 & 24.0 & 63.0 & 37 & 12.0 & 28.9 & 22.4 & 65.7 \\
\hline All rRNAs & 3,014 & 32.9 & 23.8 & 32.7 & 22.3 & 65.7 & & & & & \\
\hline All tRNAs & 1,394 & 35.4 & 12.8 & 30.2 & 21.7 & 65.6 & & & & & \\
\hline All URs & 2,681 & 28.2 & 14.1 & 34.1 & 23.6 & 62.3 & & & & & \\
\hline All genic DNA & 16,314 & 36.2 & 13.2 & 26.8 & 23.8 & 63.0 & & & & & \\
\hline All DNA & 18,995 & 35.1 & 13.3 & 27.9 & 23.7 & 63.0 & & & & & \\
\hline
\end{tabular}

\section{3}

4 


\section{Table 5 (on next page)}

Codon usage.

The codons corresponding to a tRNA present in the mitochondrial genome are underlined and in bold. The highest frequency among synonymous codons is also underlined and in bold. \# = number of codons; Frequency = frequency of each codon among synonymous codons; \%TOT =frequency of each codon among all the codons. 
1 Table 5 Codon usage. The codons corresponding to a tRNA present in the

2 mitochondrial genome are underlined and in bold. The highest frequency among

3 synonymous codons is also underlined and in bold. \# = number of codons; Frequency =

4 frequency of each codon among synonymous codons; \%TOT =frequency of each codon

5 among all the codons.

6

\begin{tabular}{|c|c|c|c|c|c|c|c|c|c|}
\hline Amino Acid & Codon & $\#$ & Frequency & \%TOT & Amino Acid & Codon & \# & Frequency & \%TOT \\
\hline \multirow{4}{*}{ Ala } & GCG & 29 & 0.15 & 0.73 & \multirow{4}{*}{ Pro } & CCG & 16 & 0.12 & 0.40 \\
\hline & GCA & 44 & 0.23 & 1.11 & & $\underline{\mathrm{CCA}}$ & 36 & 0.27 & 0.91 \\
\hline & GCT & 85 & $\underline{0.45}$ & 2.14 & & ССТ & 58 & $\underline{0.43}$ & 1.46 \\
\hline & GCC & 30 & 0.16 & 0.76 & & $\mathrm{CCC}$ & 24 & 0.18 & 0.61 \\
\hline \multirow{2}{*}{ Cys } & TGT & 94 & $\underline{0.76}$ & 2.37 & \multirow{2}{*}{ Gln } & CAG & 25 & 0.44 & 0.63 \\
\hline & $\underline{\text { TGC }}$ & 30 & 0.24 & 0.76 & & CAA & 32 & $\underline{0.56}$ & 0.81 \\
\hline \multirow{2}{*}{ Asp } & GAT & 54 & $\underline{0.66}$ & 1.36 & \multirow{4}{*}{ Arg } & CGG & 23 & 0.31 & 0.58 \\
\hline & GAC & 28 & 0.34 & 0.71 & & $\underline{\text { CGA }}$ & 21 & 0.28 & 0.53 \\
\hline \multirow{2}{*}{ Glu } & GAG & 87 & $\underline{0.6}$ & 2.19 & & CGT & 25 & $\underline{0.33}$ & 0.63 \\
\hline & GAA & 58 & 0.4 & 1.46 & & CGC & 6 & 0.08 & 0.15 \\
\hline \multirow{2}{*}{ Phe } & TTT & 269 & $\underline{0.78}$ & 6.78 & \multirow{8}{*}{ Ser } & AGG & 69 & 0.19 & 1.74 \\
\hline & $\underline{\text { TTC }}$ & 78 & 0.22 & 1.97 & & AGA & 69 & 0.19 & 1.74 \\
\hline \multirow{4}{*}{ Gly } & GGG & 131 & $\underline{0.4}$ & 3.30 & & AGT & 55 & 0.15 & 1.39 \\
\hline & $\underline{\text { GGA }}$ & 61 & 0.19 & 1.54 & & AGC & 23 & 0.06 & 0.58 \\
\hline & GGT & 98 & 0.3 & 2.47 & & TCG & 18 & 0.05 & 0.45 \\
\hline & GGC & 36 & 0.11 & 0.91 & & $\underline{\mathrm{TCA}}$ & 33 & 0.09 & 0.83 \\
\hline \multirow{2}{*}{ His } & CAT & 37 & $\underline{0.62}$ & 0.93 & & TCT & 76 & $\underline{0.21}$ & 1.92 \\
\hline & $\underline{\text { CAC }}$ & 23 & 0.38 & 0.58 & & TCC & 22 & 0.06 & 0.55 \\
\hline \multirow{2}{*}{ Ile } & ATT & 165 & $\underline{0.8}$ & 4.16 & \multirow{4}{*}{ Thr } & ACG & 21 & 0.17 & 0.53 \\
\hline & ATC & 40 & 0.2 & 1.01 & & $\underline{A C A}$ & 30 & 0.24 & 0.76 \\
\hline \multirow{2}{*}{ Lys } & AAG & 61 & 0.41 & 1.54 & & $\mathrm{ACT}$ & 57 & $\underline{0.46}$ & 1.44 \\
\hline & AAA & 87 & $\underline{0.59}$ & 2.19 & & ACC & 16 & 0.13 & 0.40 \\
\hline \multirow{6}{*}{ Leu } & TTG & 122 & 0.23 & 3.08 & \multirow{4}{*}{ Val } & GTG & 113 & 0.3 & 2.85 \\
\hline & TTA & 210 & $\underline{0.39}$ & 5.29 & & $\underline{\text { GTA }}$ & 121 & $\underline{0.32}$ & 3.05 \\
\hline & CTG & 43 & 0.08 & 1.08 & & GTT & 119 & 0.32 & 3.00 \\
\hline & CTA & 70 & 0.13 & 1.76 & & GTC & 23 & 0.06 & 0.58 \\
\hline & CTT & 75 & 0.14 & 1.89 & \multirow{2}{*}{ Trp } & TGG & 58 & $\underline{0.54}$ & 1.46 \\
\hline & СТC & 20 & 0.04 & 0.50 & & TGA & 49 & 0.46 & 1.24 \\
\hline \multirow{2}{*}{ Met } & ATG & 86 & 0.36 & 2.17 & \multirow{2}{*}{ Tyr } & TAT & 103 & $\underline{0.69}$ & 2.60 \\
\hline & ATA & 155 & $\underline{0.64}$ & 3.91 & & $\underline{\text { TAC }}$ & 47 & 0.31 & 1.18 \\
\hline \multirow{2}{*}{ Asn } & AAT & 76 & $\underline{0.66}$ & 1.92 & \multirow{2}{*}{ STOP } & TAG & 34 & 0.58 & 0.86 \\
\hline & AAC & 39 & 0.34 & 0.98 & & TAA & 25 & 0.42 & 0.63 \\
\hline
\end{tabular}




\section{Table 6(on next page)}

LUR length and number of repeats in the 13 female samples analyzed.

F3 and F17 are heteroplasmic with LURs of different length. 
1

2

3 Table 6 LUR length and number of repeats in the 13 female samples analyzed. F3 and

4 F17 are heteroplasmic with LURs of different length.

5

6

\begin{tabular}{|l|c|c|c|}
\hline Specimen & Length (bp) & Number of Repeats & GenBank Acc. No. \\
\hline F3 & $2,100-3,500$ & $6.5-25$ & MF055702 \\
\hline F5 & 5,000 & 45 & MF055703 \\
\hline F7 & 3,500 & 25 & MF055704 \\
\hline F9 & 3,500 & 25 & MF055705 \\
\hline F10 & 3,000 & 20 & MF055706 \\
\hline F11 & 3,000 & 20 & MF055707 \\
\hline F13 & 3,500 & 25 & MF055708 \\
\hline F15 & 3,000 & 20 & MF055709 \\
\hline F16 & 3,500 & 25 & MF055710 \\
\hline F17 & $2,500-3,500$ & $8-25$ & MF055711 \\
\hline F19 & 3,500 & 25 & MF055712 \\
\hline F20 & 2,500 & 8 & MF055713 \\
\hline F21 & 2,100 & 6.5 & MF055714 \\
\hline
\end{tabular}

7 


\section{Table 7 (on next page)}

Sequence Polymorphism (SP): SNPs and small indels called by GATK.

$\mathrm{CDS}=$ coding sequences; Whole $\mathrm{mtDNA}=$ polymorphism in the whole mitochondrial genome; the number in brackets the bottom of the table represent private SPs (e.g.: there are 23 female specific SPs in the whole mtDNA and 9 female specific SPs in CDS); $p$-value = significance of the Fisher's exact test on number of SPs between sexes (i.e.: all males vs females, males except mRDI01 vs females). 
1 Table 7 Sequence Polymorphism (SP): SNPs and small indels called by GATK.

$2 \mathrm{CDS}=$ coding sequences; Whole mtDNA = polymorphism in the whole mitochondrial

3 genome; the number in brackets the bottom of the table represent private SPs (e.g.:

4 there are 23 female specific SPs in the whole mtDNA and 9 female specific SPs in

5 CDS); $p$-value = significance of the Fisher's exact test on number of SPs between sexes

6 (i.e.: all males vs females, males except mRDI01 vs females).

7

8

\begin{tabular}{|c|c|c|c|c|c|}
\hline FEATURE & VALUE & MIN & MEDIAN & MEAN & MAX \\
\hline Depth (all SPs) & - & 6 & 1,357 & 1,521 & 3,880 \\
\hline Phred Score (all SPs) & - & $3.30 \mathrm{E}+01$ & $5.76 \mathrm{E}+03$ & $4.18 \mathrm{E}+07$ & $2.15 E+09$ \\
\hline Depth (SPs in CDS) & - & 222 & 2,038 & 2,150 & 3,880 \\
\hline Phred Score (SPs in CDS) & - & $1.18 \mathrm{E}+02$ & $1.01 \mathrm{E}+04$ & $4.45 \mathrm{E}+07$ & $2.15 E+09$ \\
\hline Total number of SPs & 257 & - & - & - & - \\
\hline $\begin{array}{l}\text { Number of mRDI01 private } \\
\text { SPs }\end{array}$ & $\begin{array}{l}151(58.7 \% \text { of } \\
\text { the total) }\end{array}$ & - & - & - & - \\
\hline Number of SPs in CDS & $\begin{array}{c}145(56.4 \% \text { of } \\
\text { the total })\end{array}$ & - & - & - & - \\
\hline $\begin{array}{l}\text { Number of mRDI01 private } \\
\text { SPs in CDS }\end{array}$ & $\begin{array}{l}103(71 \% \text { of the } \\
\text { SNPs in CDS) }\end{array}$ & - & - & - & - \\
\hline $\begin{array}{l}\text { Number of SPs in CDS } \\
\text { (excluding mRDI01) }\end{array}$ & 42 & - & - & - & - \\
\hline Frequency of SPs in CDS & $\begin{array}{c}0.0097(\sim 1 \\
\text { every } 103 b p)\end{array}$ & - & - & - & - \\
\hline $\begin{array}{l}\text { Frequency of SPs in CDS } \\
\text { (excluding mRDI01) }\end{array}$ & $\begin{array}{c}0.0028(\sim 1 \text { every } \\
355 \mathrm{bp})\end{array}$ & - & - & - & - \\
\hline Total number of indels & 18 & - & - & - & - \\
\hline Number of indels in CDS & 6 & - & - & - & - \\
\hline $\begin{array}{l}\text { Number of indels causing } \\
\text { frameshift }\end{array}$ & 4 & - & - & - & - \\
\hline
\end{tabular}

\begin{tabular}{lcc}
\hline \# OF SPs & WHOLE mtDNA & CDS \\
\hline Males & $234(160)$ & $136(107)$ \\
\hline Males (no mRDI01) & $84(15)$ & $32(6)$ \\
\hline Females & $97(23)$ & $38(9)$ \\
\hline
\end{tabular}


Table 8 (on next page)

Indels located in coding sequences.

DEPTH = sequencing depth; QUAL = quality of the called SNP expressed in Phred score;

ALLELE FREQUENCY $=$ frequency of the alternative allele in each sample indicated in the "SAMPLE" column. 
1 Table 8 Indels located in coding sequences. DEPTH = sequencing depth; QUAL =

2 quality of the called SNP expressed in Phred score; ALLELE FREQUENCY = frequency

3 of the alternative allele in each sample indicated in the "SAMPLE" column.

4

\begin{tabular}{|c|c|c|c|c|c|c|c|c|}
\hline POSITION & DEPTH & QUAL & GENE & SNP & FRAMESHIFT & SAMPLE & $\begin{array}{c}\text { ALLELE } \\
\text { FREQUENCY }\end{array}$ & NOTES \\
\hline 1,698 & 3,732 & $1.38 \mathrm{E}+04$ & $\operatorname{cox} 1$ & C/CAAA & No & $\begin{array}{l}\text { mRDI02, } \\
\text { mRDI03 }\end{array}$ & $0.089,0.85$ & Insertion of 1 Lysine \\
\hline 6,364 & 1,929 & $2.15 E+09$ & cytb & $\mathrm{CT} / \mathrm{C}$ & Yes & $\begin{array}{l}\text { fRDI04, } \\
\text { mRDI05 }\end{array}$ & $0.80,0.81$ & $\begin{array}{l}\text { Yields a shorter Cytb. } \\
\text { Possible sequencing } \\
\text { error due to the } \\
\text { homopolymer } \\
\text { CTTTTTTT }\end{array}$ \\
\hline 10,449 & 1,780 & $2.15 E+09$ & $n d 5$ & $\mathrm{C} / \mathrm{CT}$ & Yes & $\begin{array}{l}\text { fRDI01, } \\
\text { fRDI04, } \\
\text { fRDI05 }\end{array}$ & $\begin{array}{c}0.11,0.10 \\
0.11\end{array}$ & $\begin{array}{c}\text { Yields a nd5 gene } \\
\text { divided in } 2 \text { ORFs. } \\
\text { Possible sequencing } \\
\text { error due to the } \\
\text { homopolymer CTTTTTT }\end{array}$ \\
\hline 17,619 & 2,272 & $5.98 \mathrm{E}+03$ & $\cos 3$ & AGCG/A & No & mRDI01 & 0.97 & Deletion of 1 Alanine \\
\hline 17,621 & 2,188 & $9.99 \mathrm{E}+04$ & $\operatorname{cox} 3$ & $\mathrm{CG} / \mathrm{C}$ & Yes & mRDI01 & 0.99 & $\begin{array}{c}\text { Always combined with } \\
\text { SNP_17624. Together } \\
\text { change the last } 35 \text { amino } \\
\text { acids. }\end{array}$ \\
\hline 17,624 & 2,287 & $5.98 \mathrm{E}+03$ & $\operatorname{cox} 3$ & C/CAT & Yes & mRDI01 & 0.99 & $\begin{array}{c}\text { Always combined with } \\
\text { SNP_17621. Together } \\
\text { change the last } 35 \text { amino } \\
\text { acids. }\end{array}$ \\
\hline
\end{tabular}

